\title{
Usage Patterns of Privacy-Enhancing Technologies
}

\author{
Kovila P.L. Coopamootoo \\ Newcastle University \\ Newcastle-Upon-Tyne, UK \\ kovila.coopamootoo@newcastle.ac.uk
}

\begin{abstract}
The steady reports of privacy invasions online paints a picture of the Internet growing into a more dangerous place. This is supported by reports of the potential scale for online harms facilitated by the mass deployment of online technology and by the data-intensive web. While Internet users often express concern about privacy, some report taking actions to protect their privacy online. We investigate the methods and technologies that individuals employ to protect their privacy online. We conduct two studies, of $\mathrm{N}=180$ and $\mathrm{N}=907$, to elicit individuals' use of privacy methods, within the US, the UK and Germany. We find that non-technology methods are among the most used methods in the three countries. We identify distinct groupings of privacy methods usage in a cluster map. The map shows that together with non-technology methods of privacy protection, simple PETs that are integrated in services, form the most used cluster, whereas more advanced PETs form a different, least used cluster. We further investigate user perception and reasoning for mostly using one set of PETs, in a third study with $\mathrm{N}=183$ participants. We do not find a difference in perceived competency in protecting privacy online between advanced and simpler PETs users. We compare use perceptions between advanced and simpler PETs and report on user reasoning for not using advanced PETs, as well as support needed for potential use. This paper contributes to privacy research by eliciting use and perception of use across 43 privacy methods, including 26 PETs across three countries and provides a map of PETs usage. The cluster map provides a systematic and reliable point of reference for future user-centric investigations across PETs. Overall, this research provides a broad understanding of use and perceptions across a collection of PETs, and can lead to future research for scaling use of PETs.
\end{abstract}

\section{CCS CONCEPTS}

- Security and privacy $\rightarrow$ Human and societal aspects of security and privacy; Privacy protections; Social aspects of security and privacy; Usability in security and privacy.

\section{KEYWORDS}

Privacy; Privacy-Enhancing Technology; Usage; Perception of Use; User-Study

Permission to make digital or hard copies of all or part of this work for personal or classroom use is granted without fee provided that copies are not made or distributed for profit or commercial advantage and that copies bear this notice and the full citation on the first page. Copyrights for components of this work owned by others than the author(s) must be honored. Abstracting with credit is permitted. To copy otherwise, or republish, to post on servers or to redistribute to lists, requires prior specific permission and/or a fee. Request permissions from permissions@acm.org.

CCS '20, November 9-13, 2020, Virtual Event, USA

(c) 2020 Copyright held by the owner/author(s). Publication rights licensed to ACM ACM ISBN 978-1-4503-7089-9/20/11 . \$ \$15.00

https://doi.org/10.1145/3372297.3423347
ACM Reference Format:

Kovila P.L. Coopamootoo. 2020. Usage Patterns of Privacy-Enhancing Technologies. In Proceedings of the 2020 ACM SIGSAC Conference on Computer and Communications Security (CCS '20), November 9-13, 2020, Virtual Event, USA. ACM, New York, NY, USA, 20 pages. https://doi.org/10.1145/3372297.3423347

\section{INTRODUCTION}

The deployment of technology at massive scale is thought to have enabled the rapid emergence of a set of 'online harms' such as privacy abuses, data breaches, cyber-attacks, inappropriate uses of personal data, hate crimes, and self-harm/suicide among others [109]. Today's data-intensive web is characterized with mass sharing, collection and aggregation of individuals' data, that enables the provisioning of customised services but unfortunately also engenders targeted advertising [14], digital discrimination [31], privacy invasive algorithmic computations [35], and a general fuzzyness about privacy rights online. The recent years have indeed seen reports of various high profile privacy infringement cases involving mass unauthorised transfer and use of sensitive data [37, 70, 82].

At the forefront of the narrative that "the Internet is a dangerous place", is the UK, where the British Government has released an Online Harms white paper that elaborates on the scale and extent of harms faced by individuals online. It proposes a mission of mitigating online harms without inhibiting online innovation [109]. One of the strands of this mission is to empower citizens via technology, in particular via awareness, usage and integration of PrivacyEnhancing Technologies (PETs) in real life situations. PETs are defined by the EU Agency for CyberSecurity, as technologies shaped according to privacy principles, where PETs "covers the broader range of technologies that are designed for supporting privacy and data protection" [32]. Integrating PETs in daily life and scaling use of PETs, first requires a broad understanding of current use of PETs.

For a user perspective of online harms, the Oxford Internet Institute looked into how UK individuals' experience of problems online influence their behavior, in an Oxford Internet Survey (OxIS) [13]. They found that non-users of the Internet (who made up $10 \%$ of the respondents), were more concerned than users about privacy violations, consequently keeping themselves offline. Surprisingly, Internet users were not more concerned about the possibility of being a victim online in 2019 compared to 2013. In 2019, general privacy concern increased only by $4 \%$, and $26-31 \%$ of respondents have taken action to protect their purchases, age, marital status or medical details and $40 \%$ their contact details. According to Pew Research Center of the US, although a majority of US consumers think that they have little or no control over how their personal information is collected and used by companies ( $81 \%$ in 2019 compared to $91 \%$ in 2013) $[5,64]$, between $10 \%$ to $19 \%$ feel that they have a lot of control over different forms of personal information, such as physical location, social media posts, private conversations, 
purchases, or websites visited [5]. The European Commission reports a Eurobarometer survey in 2019 with findings that $51 \%$ of those who provide personal information online felt that they have partial control over this information, while $14 \%$ felt that they have complete control. $62 \%$ of those feeling partial or no control were concerned about not having complete control. In addition, a majority of the respondents in 2019 have heard of the rights guaranteed by the GDPR, and some have exercised these rights [34].

Given (1) the above reports of nuances in privacy concerns over the years and that some individuals take actions towards privacy, as well as (2) the well known privacy paradox phenomenon [4, $38,55,95]$, this paper seeks to investigate the following questions: 'what privacy methods or PETs do Internet users employ to protect their privacy? How do Internet users perceive the use of PETs?' We contribute to the rich landscape of user-centric privacy research, that has expansively addressed privacy behavior, including via the (extent of) disclosure of personal information [3, 4, 8, 26, 72, 95], privacy strategies $[1,17,75,105]$ or the use of privacy controls and individual PETs [1, 12, 26, 39, 46, 71, 87, 100], with a large-scale and cross-national study of the use of a collection of PETs.

We first investigate the use/non-use of a range of privacy methods and PETs across three countries, rather than taking an in-depth look at how users interact with individual PETs [12, 46, 71, 87] or engage with controls $[39,69]$. This provides a broad view of what privacy methods and PETs individuals use, and enables us to uncover patterns of use and preferences.

Second, while various factors may influence the use/non-use of particular privacy controls and PETs, such as perceived risks [36, $39]$, perceived usefulness [12, 46] or demographics [75, 76], we focus on what happens following potential concern about privacy, in particular, whether individuals are aware of PETs and how they perceive use of PETs (c.f. the staircase/steps towards using a particular PET by Renaud et al. [87]). We postulate that PETs use patterns may be evidence of differences in use perceptions, in rationale for use or support needed. Therefore, rather than investigating perception of use of individual PETs [12, 46], we evaluate perceptions across clusters of PETs. This enables us to investigate whether (1) use perceptions and rationale are clearly demarcated across the patterns, as well as (2) to simultaneously understand why individuals prefer a particular collection of PETs.

Contributions. We provide both the quantitative grounding of classification, as well as the rich qualitative investigation of why individuals prefer one type of PETs over another. We provide a large-scale, cross-national, mixed-methods (quantitative and qualitative) investigation of PETs usage. We classify patterns of use of privacy methods online (including PETs and non-technology methods), in Studies $1 \& 2$ via a clustering approach. This provides a systematic, evidence-based and reliable point of reference for PETs usage categorisation and future research, and a classification based on patterns of use of PETs (rather than design type or protection provided). We therefore also provide re-usable methodology to better understand clusters of PETs, that can further drive investigations and understanding of PETs use via comparison of clusters, transition between clusters or inter-PETs usage.

We find that the cluster map distinguishes usage of PETs into Advanced and Other PETs, and that non-technology privacy methods are among the most used methods online. We demonstrate use of the PETs usage classification within an investigation of why certain PETs are preferred over others, in Study 3. While we find support for themes identified in previous privacy controls and PETs use research, in particular with regards to awareness of privacy protection [87, 93], perceived usefulness [12, 46], usability [1, 87], or trust $[12,46]$, we expand on the themes of information about PETs, privacy needed and usability as well as report other rationales for not using advanced PETs, such as perceived monetary cost and social support. We also find that there is no statistical difference in individuals' perceived competency to protect their privacy online, whether they are Advanced or Other PETs users.

Outline. The rest of the paper is organised as follows: we first review background research, and then provide a section describing Study 1 and 2, via their aim, methodology and results. We proceed into presenting Study 3, also via aim, methodology and results. We complete the paper with an overall discussion and conclusion, and provide an Appendix with additional support, including the questionnaires used in the studies.

\section{BACKGROUND}

Given our paper computes patterns of privacy methods usage and investigates user perceptions across clusters, we provide a review of literature addressing (1) privacy behavior, of which use of PETs is one aspect, and (2) technology adoption and classification of user responses in privacy research.

\subsection{Privacy Behavior}

We review a few strands of privacy behavior related research, in particular, (1) different protection practices, (2) factors impacting use of privacy controls and PETs, and (3) cross-national investigations of privacy concerns and behavior.

2.1.1 Privacy Protection Practices. The privacy research community is well acquainted with the privacy paradox phenomenonwhere on the one hand users express concerns about the handling of their personal data and desire protection, while on the other hand, they voluntarily disclose via social networks or rarely make an effort to protect their data actively [3, 4, 15, 26, 72, 95]. Observations of the privacy paradox have also been discussed [26, 81] and systematic reviews of privacy paradox research conducted [9, 38, 55]. One of the resulting observations is that both privacy attitude and behavior can be conceptualised in different ways $[20,38,55]$.

Research has operationalised privacy behavior in different ways, including via (the extent of) disclosure, via use of privacy controls and PETs, or via the adoption of protection strategies. First, privacy behavior as disclosure include observations such as revelations to an online bot [95], Facebook membership [3] and revelations in a bank and pharmaceutical scenario [72] or self-reports such as usage of Facebook or information disclosed $[4,8,26]$. Second, research has also operationalised privacy behavior as engaging in protective control actions and using privacy technology including the general use of privacy controls [39], use of controls in the context of social networks $[26,100]$, and use of secure encrypted communication [1, 87], anonymous credentials [12], anonymity service [46], and VPN [71], or reading the privacy policy [99]. Third, protection strategies have been investigated such as providing incorrect [75] or false [17] information, delivering sensitive information in-person 
Table 1: Factors Influencing Protective Privacy Behavior

\begin{tabular}{ll}
\hline Factor & PETs/Context of Use \\
\hline Years of internet experience & social/technical protection [76] \\
Internet skill & Facebook privacy settings [47] \\
Perceived rewards in disclosure & general/technical protection [69] \\
Privacy risks concerns & general/technical protection [69] \\
Unawareness of risks & social networks [39] \\
Risks of sharing & social networks [36] \\
Perceived usefulness \& ease of use & anonymizing technology [12, 46] \\
Emotional considerations & VPN use [71] \\
Knowledge about how to protect oneself & E2EE [87] \\
Awareness of protection tools & E2EE \& tracking [87, 93] \\
No perceived need to act & E2EE \& tracking [87, 93] \\
Inability to use protection & tracking [93] \\
Becoming side-tracked & tracking [93] \\
Usability & E2EE \& secure msg [1, 87] \\
Social Influence & secure msg \& social networks [1, 39] \\
Education & Facebook sharing [36] \\
Gender & technical protection [75, 76], \\
& social network control [98] \\
\hline \multicolumn{1}{c}{ Note: E2EE refers to end-to-end encryption }
\end{tabular}

or using a "code" to deliver sensitive information to others [1], and deleting friends or rejecting friend requests, self-censoring or deleting content on social network [105].

2.1.2 Factors Influencing Protective Privacy Behavior. Various research have pointed to factors affecting protective control actions including a systematic review with comparison of predictor effect sizes [38], and obstacles to adoption of PETs [39, 87, 93] and secure communication [1]. We provide a summary of factors in Table 1 (focusing on use of controls and PETs as behavior, rather than disclosure or protection strategies described above in Section 2.1.1). We also name the context of investigation, such as social networks for inbuilt PETs or name the standalone PET investigated. We note that some factors were found to be more important than others, for example the risk of sharing [36], awareness of consequences of privacy violations [39] and contextual aspects of the messaging tool such as fragmented user bases [1], were found to be more important than usability.

2.1.3 Cross-National Influence. While there is no universal privacy or data protection law that applies across the whole Internet, a number of international and national privacy frameworks have largely converged to form a set of core, baseline privacy principles, that establish fair information practices for consumers, businesses and organisations. Whereas the General Data Protection Regulation (GDPR) provides the legal backbone for data protection and privacy in Europe [104], the US does not have an all-encompassing law like the GDPR but has a variety of federal and state laws that aim to protect a citizen's privacy and online data [79] and the UK established the Data Protection Act 2018 as its implementation of the GDPR [77]. With regards to Internet users, differences have been observed in privacy concern, behavior or valuation of information across countries [10, 16, 22, 65, 86, 97], with some influence of national culture (where national culture is viewed as the collective mindset distinguishing members of one nation from another [48], and is embedded in the way members think, feel and act [49]), in particular via the collectivist versus individualist distinctions [16, 65, 86] or relational mobility [97]. Others have looked into the privacy calculus across countries in contexts such as ecommerce [28], social network [56], health records [27] or driving behavior [54]. These studies leveraged population samples worldwide [10, 86], or compared the US versus Asian countries [65, 97], the US versus Asia-Pacific countries [16], the US versus European countries [27, 28, 54, 56], or across European countries [22, 44].

\subsection{Technology Adoption \& Patterns}

2.2.1 Model of Technology Adoption. The Technology Acceptance Model (TAM) provides the theoretical background for understanding why users accept or reject technology [24]. It has been empirically demonstrated to successfully predict $40 \%$ of system use [60]. TAM suggests that perceived ease of use (PEOU) and perceived usefulness (PU) are two most important factors explaining technology use. Privacy research employing TAM as psychological construct ranged from (1) understanding adoption of specific PETs, such as user acceptance of anonymous credentials [12], anonymity technology [46], and VPNs [71]; to (2) perceived privacy in adoption of technologies such as social media [84], e-commerce [57, 103], biometrics [68], Snapchat [61] or trading systems [89].

2.2.2 Cluster Analysis for User-Centric Privacy. Investigations into patterns of use of technology may enable identification of broad usage trends across single or a collection of technologies. This can be conducted via cluster analysis, which supports the identification of patterns and provides an objective methodology for quantifying structural characteristics of observations, and relationship identification [7, 63]. Cluster analysis has also been employed in user-centric studies in the privacy context to better understand perceptions or behaviors. Example investigations using cluster analysis include SNS engagement and privacy habits in relation to transport [30], privacy management strategies in Facebook [59], privacy perceptions based on geographical regions [50], perceptions of information sensitivity between countries [92], cross-country comparison of adolescents' privacy perceptions [94], privacy versus sharing perceptions [74], and privacy and risks perceptions to better understand behavior [21].

\section{STUDY $1 \& 2$}

\subsection{Aim}

We explore usage of privacy methods online in visual maps. We sample participants from the US, UK and German populations.

3.1.1 Privacy Method Patterns. We posit that individuals likely engage with privacy via a habitual collection of methods or pattern of actions, where habits are actions that have become automatically triggered by situational cues [58]. We investigate as RQ1, "What patterns emerge in individuals' privacy methods usage and preference? How similar are usage patterns across privacy methods?" Because we do not have a-priori expectations of the nature of relationships between privacy method preferences, we employ a Cluster Analysis [63] to naturally distinguish different patterns of use, and further look into usage patterns in relation to country via a Correspondence Analysis [40, 41].

3.1.2 Cross-National Privacy Method Similarities. The diffusion and adoption of technology do not necessarily follow a common pattern in terms of rates or timing across countries. Adoption of technology is often influenced by cross-national factors [6, 33], 
where in particular, use of technology for privacy may be influenced by regulation. We investigate as RQ2, "What methods are mostly used to protect one's privacy online? what similarities emerge between countries?"

\subsection{Method}

We conduct two survey studies online. The first study (Study 1) is aimed at identifying a preferred list of privacy methods. The second study (Study 2) employs the compiled list of methods from Study 1 to query participants about their use of the range of privacy methods identified.

3.2.1 Participants. With their advanced digital economies, Europe and the US may be considered as the drivers of protection technology around the globe. However, Europe versus the US differ in privacy regulation [83] and potentially also in individuals' privacy protection patterns.

For Study 1, we sampled $N=180$ participants, comprising $N=$ 58 US participants, $N=62$ UK participants and $N=60$ German (DE) participants. We sampled participants from Prolific Academic. The data quality of Prolific Academic has good reproducibility [78], and is comparable to Amazon Mechanical Turk's which is widely used in security and privacy user studies.

For Study 2, we recruited an $N=907$ sample from the US, UK and DE via Prolific Academic. The samples from the US and the UK were representative of age and gender of the respective countries, as provided by Prolific Academic. For the DE sample, we did not achieve a representative sample in terms of gender and age.

Table 2 provides a summary of the demographic details for the two studies. The studies lasted between 10 to 20 minutes. Participants were compensated at a rate of $£ 7.5$ per hour, slightly above the minimum rate of $£ 5$ per hour suggested by Prolific Academic.

Table 2: Participant Characteristics

\begin{tabular}{|c|c|c|c|c|c|}
\hline & \multirow[t]{2}{*}{ Country } & \multirow[t]{2}{*}{$N$} & \multirow[t]{2}{*}{ Mean Age } & \multicolumn{2}{|c|}{ Gender } \\
\hline & & & & \#Female & \#Male \\
\hline \multirow{3}{*}{ Study 1} & US & 58 & 35.53 & 29 & 29 \\
\hline & UK & 62 & 30.65 & 43 & 19 \\
\hline & $\mathrm{DE}$ & 60 & 30.68 & 27 & 33 \\
\hline \multirow{3}{*}{ Study 2} & US & 303 & 43.72 & 155 & 148 \\
\hline & UK & 303 & 44.21 & 154 & 149 \\
\hline & DE & 301 & 28.91 & 115 & 186 \\
\hline
\end{tabular}

3.2.2 Procedure. Study 1 aims to identify and compile a list of privacy methods preference. We do so via an open-ended question, across three countries. Study 1 consisted of a questionnaire on demographics, and an open-ended query to list three to five tools most often employed to achieve the purpose of privacy online.

Study 2 followed the same format as Study 1, except that we changed the open-ended queries of the first study to close-ended privacy methods questions, for participants to select the methods they mostly use from the whole list provided. We also shifted to a larger sample for the three countries. We provide a summary of the procedure in Figure 1.

3.2.3 Measurement Apparatus. Study 1: We queried participants on the individual privacy methods they most often use, eliciting their own methods via the instruction "List between 3 to 5 tools

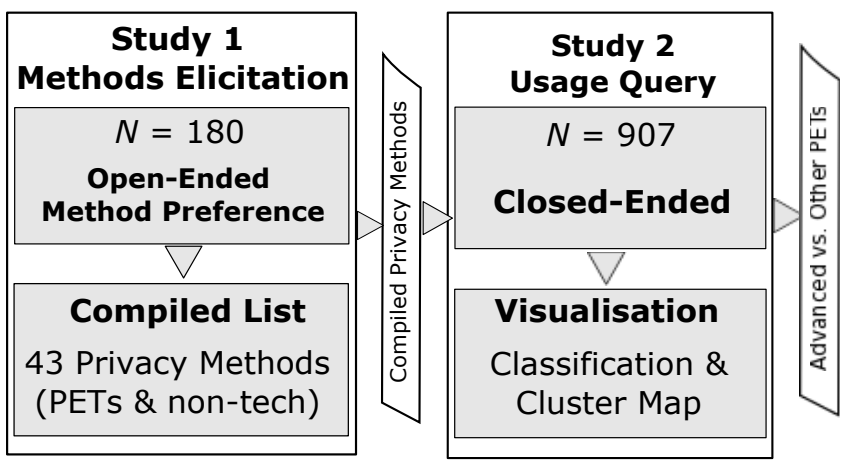

Figure 1: Studies $1 \& 2$ design.

that you have most often employed before to achieve the purpose of privacy online."

Study 2: We asked participants to rate the list of privacy methods provided with whether they use them 'very often' or 'very rarely/not at all'. While we opted for binary data, we found that querying 'If you have used them before: Yes/No' would not fit our inquiry. 'Yes' would include 'once and never again' usage type. We wanted to capture methods that are popular with users (and include protections that are often but not always used by choice) via 'very often' versus those participants have not thought of or have used in very rare circumstances (not the go-to protection).

We provide the full question and wording as provided to participants in Table 12 in the Appendix A. We pre-tested this list for comprehension with $N=7$ colleagues and acquaintances in the computing and social science departments, and believe that participants in Study 2 understood the wordings, as they were named by participants in a similar sample in Study 1 . We also provide an open-ended option for 'other privacy method'.

We encouraged participants to respond truthfully by stating that the surveys are anonymous in the consent form, and also asking for truthful answers in the questionnaires. We included attention check questions throughout the survey and the list of privacy methods presented to participants in Study 2 was randomised.

3.2.4 Limitations. Representative Sample: While in Study 2 our sample is representative of the US and UK populations, with respect to age and gender, we did not achieve a representative sample for DE (Germany). This explains the difference in mean age for DE compared to the US and UK, in Table 2. From the 2019 demographics record, the German population has a median age of 47.4, with $37.3 \%$ of the population aged over 54 [51]. The crowd sourcing platform could not cater for the representative sample for the sample size we required. Our relatively large sample size however decreases the probability of assuming as true a false premise. In addition, our sample characteristic is likely transferable to the larger German population, where participants in our sample exhibit behaviors of the general population [80]. However, in future research, it will be valuable to strive towards representativeness across the sample, including via attributes such as ethnicity and education level. Alternative crowdsourcing platforms for participant recruitment may also need to be investigated.

Self-Report Bias: Our studies rely mainly on self-reports rather than system observations. Self-report is a valuable and widely used 
Table 3: Privacy Methods Categorised by Design Type and Privacy Protection (as elicited in Study 1). We provide the shorthand shown in the Cluster Map in brackets, where applicable.

\begin{tabular}{|c|c|c|c|c|}
\hline \multirow{23}{*}{ Protection } & & & Design Type & \\
\hline & & Built-in & Standalone & Non-Technology \\
\hline & Anonymity & Encryption & Erasery & Not Store Info (notstore) \\
\hline & & Clear/Delete info/history (clearhistory) & Tor & Anonymous profile names (anonprofile) \\
\hline & & Pseudonyms/Onion (pseudonyms) & Proxy & NotGivePI / LimitSharing / MinimalInfo (limitshare) \\
\hline & & & IPHider & Several/Bogus / LimitedUse Emails (afewemail) \\
\hline & & & Virtual machine (virtualma) & Fake Info \\
\hline & & & & Limit Use of SNS Accounts (limitsns) \\
\hline & & & & SwitchOffCamera/Devices/PortableHD (switchoffcam) \\
\hline & & & & No Access Acc In Public Place/Networks (nopubac) \\
\hline & & & & Not use FB (noFB) \\
\hline & & & & Not Engaging Online/Careful/Not Signing Up (not-engage) \\
\hline & Browsing History \& & Private Browsing/incognito (privbrowsing) & DuckDuckGo & \\
\hline & Tracking Prevention & Anti-tracking addon (antitrack) & Ghostery & \\
\hline & & No location tracking (nolocation) & NoScript & \\
\hline & & Clear/Limit cookies (clearcookies) & & \\
\hline & Communication \& & Adblock & Firewall & \\
\hline & Filtering & HTTPS & VPN & \\
\hline & Prevent Leaking \& & Privacy settings (privset) & Password manager (pwd mger) & Not save (notsavepwd) or reuse password (notreusepwd) \\
\hline & Stealing of Data & Opt out & Paypal & Read terms of service (readterms) \\
\hline & & Private profiles (anonprofile) & Anti-spyware (antispy) & Request data collected, GDPR (reqdatacol) \\
\hline & & & Anti-malware (antimal) & no newsletter, think twice (nonewslet) \\
\hline & & & Kapersky & Website care/No suspicious sites (suspiciousweb) \\
\hline
\end{tabular}

method of querying users in security and privacy user studies. While self-reports can be argued to induce bias, research investigating response bias in security user studies has found that self-report insights can translate to real-world environments [85].

The list of privacy methods presented to participants in Study 2 were named from a pool of participants of $N=180$ across the three countries in Study 1. We pre-tested the list for comprehension for Study 2 with researchers who may be thought to bias the test outcomes. However, the list was sourced from participants themselves in Study 1, therefore came from the 'field'.

3.2.5 Ethics. We obtained approval from the University's Faculty Ethics Committee before starting the research, for all the studies (Studies 1 \& 2, as well as Study 3). We sought participants' consent for data collection prior to their responding to the questionnaires, and we did not collect identifying information. Participants were free to leave the survey at anytime and were compensated slightly above the advised rate as described in Section 3.2.1.

\subsection{Results}

3.3.1 Compiled Privacy Methods from Study 1. We collect participants' responses of 3 to 5 most used privacy tools or methods in Study 1 , with the $N=180$. We end up with 43 privacy methods coded across the three countries and 11 participants stating they 'don't know'.

Participants reported privacy methods that may be designed as (a) a standalone privacy technology, or as (b) privacy controls or settings integrated (built-in) within other tools such as browsers or messaging tools. Participants also reported strategies that do not involve using privacy controls, such as 'give fake info', which we name as 'non-technology' methods. We also loosely name four possible protection categories, namely (1) anonymity (ANO), (2) browsing history and tracking prevention (BHP), (3) communication privacy and filtering (COP), and (4) preventing leaking and stealing of data (PLS). We present the privacy methods elicited according to its design type and privacy protection type provided, as depicted in Table 3. (Note that this loose categorisation only serves to better present the types of privacy methods named, and do not affect the rest of the analysis.)

3.3.2 Privacy Method Patterns from Study 2. We investigate RQ1, "What patterns emerge in individuals' privacy methods usage and preference? How similar are usage patterns across privacy methods?" Our dataset is a contingency table of 43 rows (privacy methods) and 3 columns (count of participants in the US, UK and DE who reported to 'very often' use the privacy method). We provide the dataset in Table 13 in Appendix B.

We conduct two multivariate analyses and visualise our dataset: (1) via a Cluster Analysis [53, 90] that classifies the set of 43 privacy methods as suggested by natural groupings in the data themselves, and produce a cluster map as a simplified depiction of the relationships between privacy methods; and (2) via a Correspondence Analysis [40,42] that investigates and visualises the relationship between the 43 privacy methods across 3 countries, as well as the similarities between privacy methods given their country profiles. The main results for this subsection are Figures 4 and 5 .

Cluster Analysis. In the next few paragraphs, we report on the cluster analysis and results via the following steps:

(1) we conduct a dimensionality reduction,

(2) we determine the optimum number of clusters and compute the cluster analysis,

(3) we assess the internal validity for the cluster analysis, and

(4) we visualise the cluster map and interpret the dimensions.

We use the R package Factoextra [52] for the cluster computation and visualisation. 
We first compute a Principal Component Analysis (PCA) [108] as dimensionality reduction technique, using the prcomp function in $\mathrm{R}$. The PCA operates on the 3 country variables to output 3 factors or principal components (PC), namely PC1, PC2 and PC3. PC1 accounts for $95.4 \%$ of the variation in the data, while PC2 accounts for $4 \%$ of variation and PC3 accounts for $0.5 \%$. We describe the importance of the principal components in Table 4. Together, PC1 and PC2 account for $99.5 \%$ of the variation in the data, which is a large amount of variance. We therefore focus on PC1 and PC2.

Table 4: Importance of Principal Components.

\begin{tabular}{lrrr}
\hline & PC1 & PC2 & PC3 \\
\cline { 2 - 4 } Standard deviation & 1.692 & 0.348 & 0.128 \\
Proportion of Variance & 0.954 & 0.040 & 0.005 \\
Cumulative Proportion & 0.954 & 0.995 & 1.000 \\
\hline
\end{tabular}

We report the factor loadings for PC1 and PC2 in Table 5, where the country variables load similarly to $\mathrm{PC} 1$, while the US and the UK load in opposite direction to DE for PC2.

Table 5: Factor Loadings.

\begin{tabular}{lrr}
\hline Variables & PC1 & PC2 \\
\hline US & 0.585 & -0.303 \\
UK & 0.580 & -0.492 \\
DE & 0.567 & 0.816 \\
\hline
\end{tabular}

Second, we employ $k$-means as cluster analysis method on the principal components. $k$-means clustering is the most commonly used unsupervised machine learning algorithm for partitioning a given data set into a set of $k$ groups or clusters [63], and groups observations by minimizing Euclidean distances between them. Our cluster analysis focuses on the first two principal components that explain $99.5 \%$ variance, and visualise them as Dimension 1 (Dim1) and Dimension 2 (Dim2), where in general, dimensions are variables or features of data objects [42]. Dim1 refers to PC1, while Dim2 refers to PC2.

To determine the optimal number of clusters, we use the elbow method, which consists of optimising the within-cluster sum of squares (WSS) [42]. We choose a number of clusters so that adding another cluster does not improve the total WSS much better. From the plot in Figure 2, the total WSS does not show a sharp improvement after 3 clusters. We subsequently conduct a $k$-means cluster analysis with 3 clusters, and summarise distances between pairs of clusters in Table 6. The 3 clusters correspond to 3 distinct groups of privacy methods preference, of sizes 19, 13, and 11. Privacy methods in the same cluster are as similar as possible in terms of user preference, with low intra-class variation of $11.7 \%$, whereas privacy methods in different clusters are as dissimilar as possible, with high inter-class variation of $88.3 \%$. We provide further cluster statistics in Table 7.

Third, for internal validation of the cluster partitions [62], we conduct a Silhouette Analysis which measures how well an observation is clustered and estimates the average distance between clusters [91]. The silhouette plot in Figure 3 displays a measure of how close each point in one cluster is to points in the neighbouring

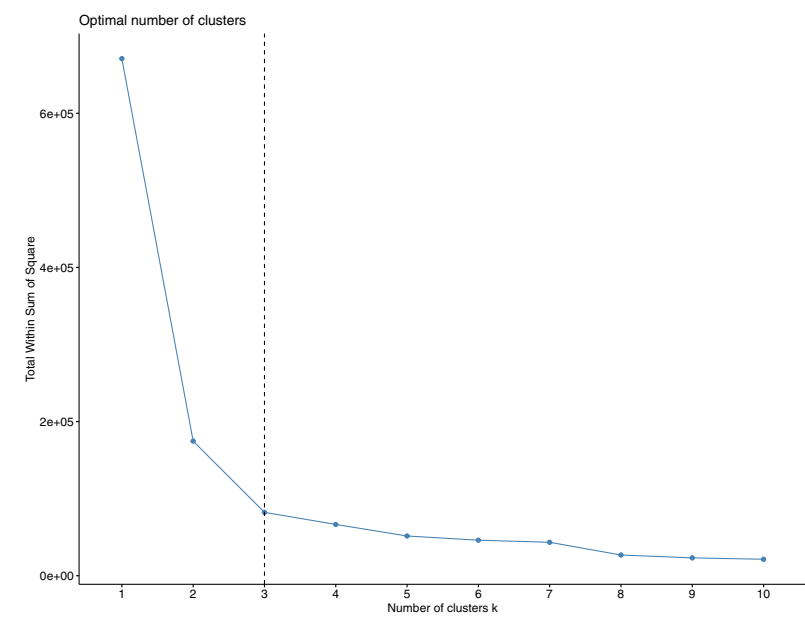

Figure 2: Determine number of clusters via Elbow Method

Table 6: Separation Matrix, that is a matrix of separation values between all pairs of clusters.

\begin{tabular}{lrrr}
\hline Cluster & 1 & 2 & 3 \\
\hline 1 & 0.0000 & & \\
2 & 160.7016 & 0.00000 & \\
3 & 48.9183 & 38.48376 & 0.00000 \\
\hline
\end{tabular}

Table 7: Cluster Statistics

\begin{tabular}{lrrr}
\hline Value Description & Cluster 1 & Cluster 2 & Cluster 3 \\
\hline Cluster size & 19 & 13 & 11 \\
Diameter & 122.69 & 115.90 & 134.38 \\
Cluster separation & 48.92 & 38.48 & 38.48 \\
Cluster avg. silhouette width & 0.58 & 0.64 & 0.43 \\
\hline Avg. distance b/w clusters & 204.53 \\
Avg. distance within clusters & 57.74 \\
Within cluster sum of squares & 82868.96 \\
Between cluster sum of squares & \multicolumn{3}{c}{623240.80} \\
\hline \multicolumn{2}{c}{ Diameter = maximum within cluster distance }
\end{tabular}

Cluster separation = vector of clusterwise minimum distances of a point in the cluster to a point of another cluster.

cluster, with an average silhouette width $S_{i}$ of 0.56 across the 3 clusters. $S_{i}$ values can range from -1 to 1 , where negative values signify that observations are placed in the wrong cluster and values close to 1 signify that observations are well clustered. Our $S_{i}$ value of +0.56 shows that our clustering is okay.

Fourth, we visualise the cluster analysis on a two-dimensional Cluster Map, as provided in Figure 4 (using the fviz_cluster function in R). Dim1/PC1 explains $95.4 \%$ of variation in the data and have similar contribution from the three countries as shown by PC1 in Table 5 and the $x$-axis of Figure 4 . The high variance explained by this factor likely represent the main characteristic of our dataset, that is the usage counts. In particular, Figure 4 shows that 'erasery' and 'suspiciousweb' are at the extreme ends of the $x$-axis and from Table 13, they are the least and most used method, respectively. We therefore interpret Dim1 to characterise 'The Popularity of Privacy 


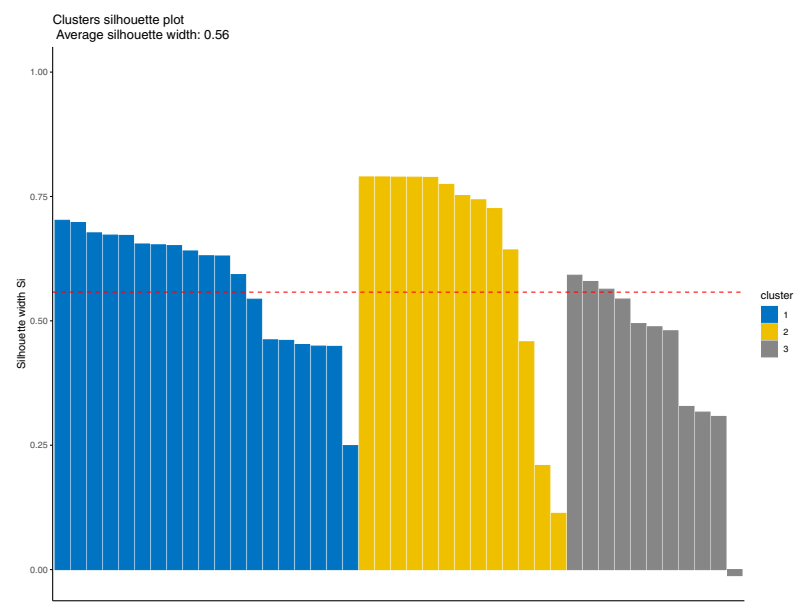

Figure 3: Silhouette Plot for Cluster Validity

Method' (both visually and confirming with the dataset). The red, right-most cluster pertains to most used privacy methods while the green and left-most cluster pertains to the least used privacy methods.

Dim2 (the $y$-axis of Figure 4, from PC2 in Table 5) depicts the country of use, where the positive end of the $y$-axis refers privacy methods usage more associated to DE, while negative end refers to usage more associated to the US and UK. For example, 'pseudonyms' and 'readterms' are both in the same cluster but are at the opposite ends of the $y$-axis. From Table 13, we find that 'pseudonyms' is most used in DE compared to US/UK while 'readterms' is least used in DE compared to US/UK.

Correspondence Analysis. The Correspondence Analysis (CA) is an exploratory technique that graphically represents the relations among rows and columns of a contingency table, in a spatial map, and is increasingly popular for dimensional reduction and perceptual mapping [42]. We use the dataset of privacy methods count across each country as in Table 13. In the next few paragraphs, we report on the $\mathrm{CA}$ and results via the following steps: (1) we compute the CA, (2) we visualise the spatial plot, and (3) we interpret the dimensions.

First, we identify 'DuckDuckGo' as an outlier, and treat it as a supplementary row, a practice used to treat outliers in correspondence analysis [11]. We compute the CA via the $C A$ function from the Factoextra package [52] in R. We find significant independence of variables with $\chi^{2}=317.016, p<.001$, and that the first dimension accounts for $92.16 \%$ of the variance in the data while the second dimension accounts for $7.84 \%$. Together these two dimensions account for $100 \%$ of variability.

Second, we visualise a spatial plot, using the fviz_ca_biplot function in R, as provided in Figure 5. The plot shows the row and column profiles simultaneously in common space. The distance between data points of the same type (row to row, that is privacy method to privacy method) is related to the degree to which the rows have similar profiles in the columns, that is relative frequencies in country variable. The more points belonging to the same set (pattern) are close to each other and the more similar their profiles are. As example, individuals who use IPHider and those who give fake information as privacy methods have similar country profiles, whereas those who use Tor and 'read terms and conditions' as privacy methods have very dissimilar country profiles.

Third, to interpret the dimensions, we look into the privacy methods and country that contributes most to defining and characterising each dimension. We provide a plot of the contribution of the privacy methods to the first dimension in the Appendix $\mathrm{C}$ as Figure 7. The top 10 privacy methods contributing to the definition of Dimension 1 (the x-axis of Figure 5) in decreasing rank, with positive (+ve) or negative (-ve) contributions are: (1) NoScript (-ve), (2) pseudonyms (-ve), (3) read terms and conditions (+ve), (4) Tor (-ve), (5) no public access (+ve), (6) virtual machine (-ve), (7) VPN (-ve), (8) not engage (+ve), (9) Ghostery (-ve), (10) give fake info (-ve). In addition, with regards to country contribution, DE contributes $61 \%$ to the definition of Dimension 1 . We interpret Dimension 1 to characterise 'Radicalness of Protective Method' ranging from the extreme positive end of the $\mathrm{x}$-axis with 'Not Engage', 'No Public Access' and 'Read terms and conditions' as non-technological solutions to the extreme negative end of the x-axis with NoScript, pseudonyms, Tor, virtual machine, VPN, Ghostery as PETs and not engaging with 'Give Fake Information'. By our interpretation, the 'Most Radical Protective Methods' are at the extreme ends of the $\mathrm{x}$-axis thereby contributing most positively and negatively, and the 'Least Radical Protective Methods' are nearer to origin $(x=z e r o)$.

We note the difference between the cluster and spatial maps. While the cluster map shows the natural grouping of privacy methods usage, the spatial map shows similarities in privacy methods usage based on their relationship to the different countries. In addition, the cluster analysis uses euclidean distance, whereas the correspondent analysis uses chi-square statistic. Therefore, Dimension 1 of both maps do not refer to the same characteristic of privacy method usage.

3.3.3 Cross-National Method Use Similarities. From Study 2, we investigate RQ2 "What methods are mostly used to protect one's privacy online? What similarities emerge between countries?" Table 8 shows a depiction of the top 10 privacy methods preferences across the three countries, where we observe that 4 of the privacy methods appear in the top 10 most reported methods in all three countries. These methods are (1) privacy settings, (2) limit sharing, (3) website care, and (4) no newsletter. In addition, we find 8 privacy methods similarities in the top 10 most reported methods for both the UK and US, 6 methods similarities between the UK and DE, and 5 methods similarities between the US and DE.

\section{STUDY 3}

\subsection{Aim}

While individuals are generally reported to be concerned about their privacy $[5,13,34,64]$, they are not necessarily observed to actively use PETs, as a consequence of their concern. In particular, a number of intervening steps may influence the 'privacy concern - use of PETs' link, such as whether individuals perceive a need to act, are aware of the usefulness PETs or are able to use PETs [87], amongst the various other factors impacting behavior listed in Table 1.

We aim to understand use perceptions and reasons for choosing a collection of PETs. Using the clusters visualised in the cluster map of Figure 4 (Study 2) as classification of privacy methods usage 


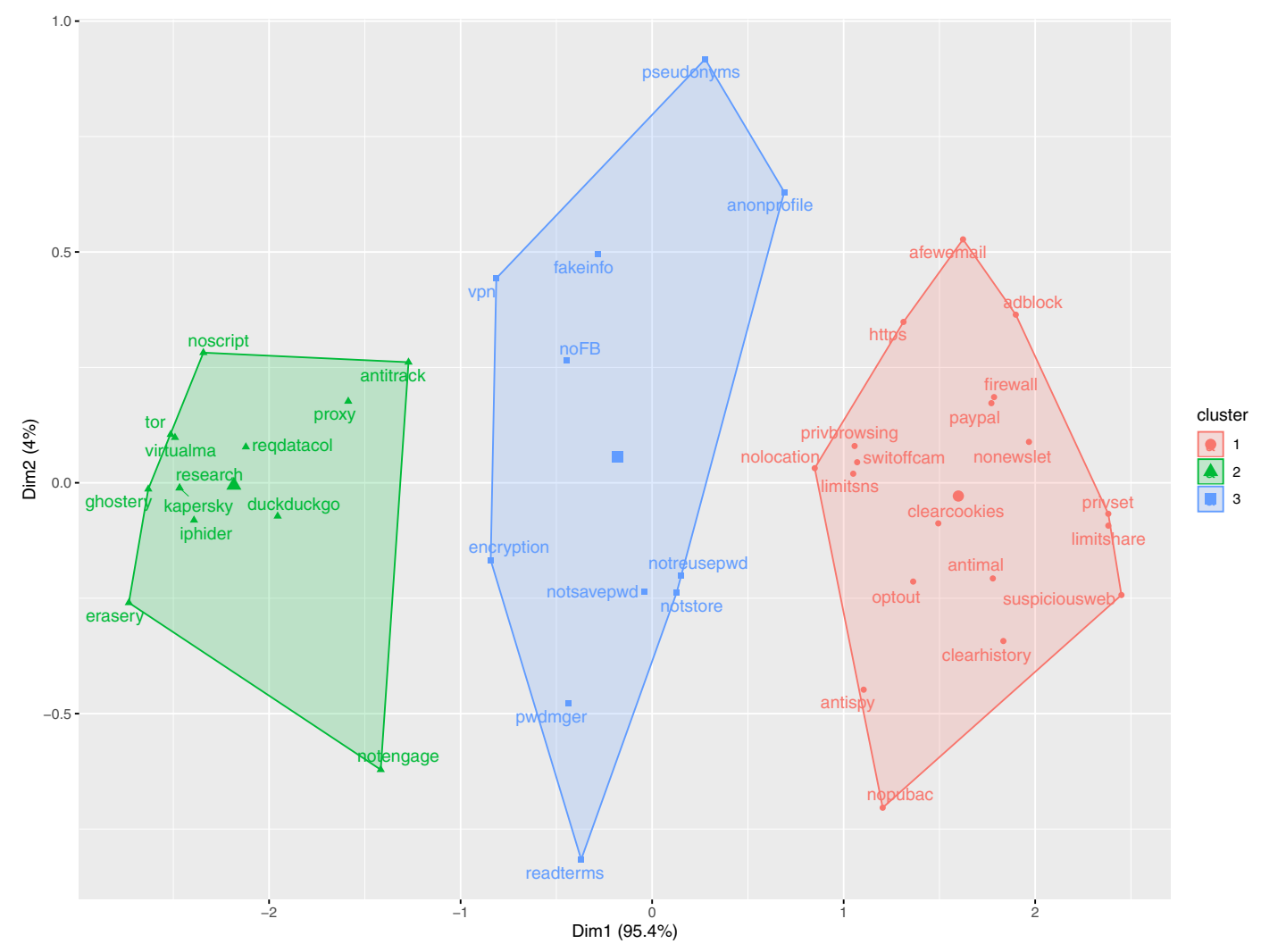

Figure 4: Privacy Methods Clusters along the Popularity of Privacy Method Dimension; left to right $=$ least to most used.

Table 8: Top 10 Privacy Methods by Country starting with most frequently mentioned

\begin{tabular}{|c|c|c|c|c|c|c|c|c|}
\hline \multicolumn{3}{|l|}{ United States } & \multicolumn{3}{|l|}{ United Kingdom } & \multicolumn{3}{|c|}{ Germany } \\
\hline Method & Design & CAT & Method & Design & CAT & Method & Design & CAT \\
\hline 1 Website care & NT & PLS & 1 Website care & NT & PLS & 1 AdBlock & BI & COP \\
\hline 2 Privacy settings & $\mathrm{BI}$ & PLS & 2 Limit Sharing & NT & ANO & 2 Bogus Emails & NT & ANO \\
\hline 3 Limit Sharing & NT & ANO & 3 Privacy settings & BI & PLS & 3 Privacy settings & BI & PLS \\
\hline 4 Research before engaging & NT & ANO & 4 Clear Info/History & $\mathrm{BI}$ & ANO & 4 Limit Sharing & NT & ANO \\
\hline 5 Anti-Malware & ST & PLS & 5 Paypal & ST & PLS & 5 No Newsletter & NT & PLS \\
\hline 6 No Newsletter & NT & PLS & 6 Research before engaging & NT & ANO & 5 Paypal & ST & PLS \\
\hline 7 AdBlock & BI & COP & 7 No Newsletter & NT & PLS & 5 Website care & NT & PLS \\
\hline 8 Clear Info/History & $\mathrm{BI}$ & ANO & 8 Firewall & ST & $\mathrm{COP}$ & 5 Firewall & ST & $\mathrm{COP}$ \\
\hline 9 Clear/Limit Cookies & BI & BHP & 9 Anti-Malware & ST & PLS & 9 HTTPS & $\mathrm{BI}$ & COP \\
\hline 10Not Access Accts in Public Place & NT & ANO & 10Not Access Accts in Public Place & NT & ANO & 10Pseudonyms & $\mathrm{BI}$ & ANO \\
\hline
\end{tabular}

$A N O, B H P, C O P \& P L S$ refer to privacy protection categories of anonymity, browsing history and tracking prevention, communication privacy \& filtering, and preventing leaking \& stealing of data respectively.

patterns, we examine individuals' perceptions of the PETs in the different clusters, and their rationale for a particular choice. We create two lists of PETs from the cluster map, that we name Advanced PETs (Adv.PETs) and Other PETs (Oth.PETs):

Advanced PETs List: Adv.PETs is populated with the PETs in the leftmost cluster, which contains the least used and more advanced solution to privacy protection. We also add VPN and encryption, which fall on the left of the centroid of the middle cluster, to the list. In particular, Adv.PETs refers to a list of the following PETs: Erasery,
Ghostery, virtual machine, Tor, NoScript, IPHider, Kaspersky, DuckDuckGo, proxy, anti-tracking extension, VPN, and encryption.

Other PETs List: Oth.PETs is populated with the PETs in the rightmost cluster, which contains the most used privacy methods. We also add pseudonyms and 'anonymous profile', which fall on the right of the centroid of the middle cluster, to the list. Oth.PETs refers to the following list of PETs: switch off location tracking, private browsing, HTTPS, anti-spyware, opt-out (of data collection), 


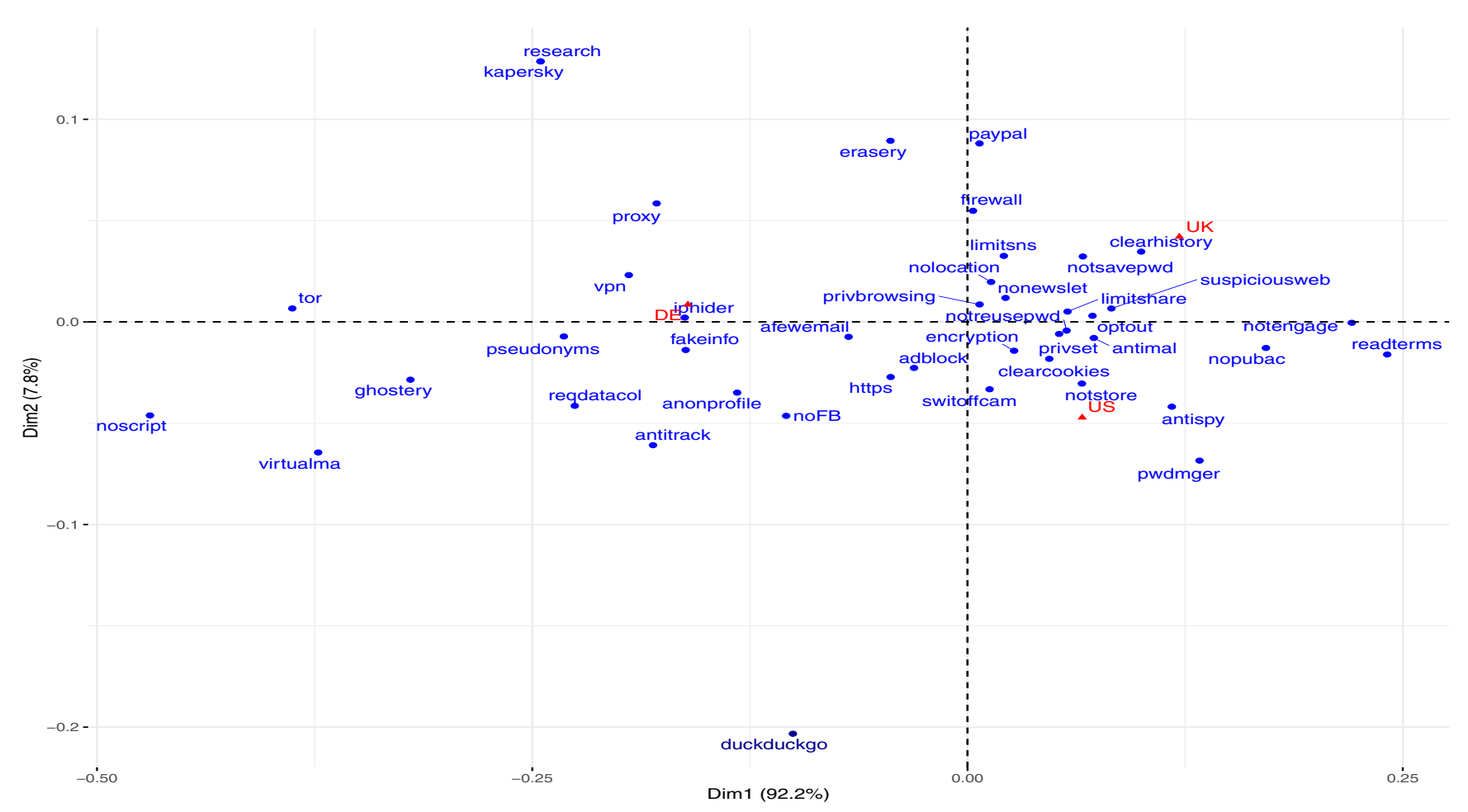

Figure 5: Spatial Plot of Privacy Methods \& Country.

clear cookies, anti-malware, clear history, Paypal, firewall, Adblock, privacy settings, pseudonyms and anonymous profile.

Research Questions. The Technology Acceptance Model (TAM), as discussed in Section 2.2.1 depicts how perceptions explain use of technology [24]. We focus on the main components of the TAM, namely perceived usefulness (PU), perceived ease of use (PEOU) as well as behavior intention (BI), to investigate perceptions of use of Adv.PETs and Oth.PETs.

We first ask "Do individuals prefer to use Adv.PETs (Adv.Users) or Oth.PETs (Oth.Users)?" We then investigate as RQ3, "How do Adv.Users' perceptions of use differ between Adv.PETs and Oth.PETs?" and "How do Oth.Users' perceptions of use differ between Adv.PETs and Oth.PETs?" via the hypotheses:

$\mathrm{H}_{3 \mathrm{a}, 0}$ : Adv.Users show no difference in perceptions of use between Adv.PETs and Oth.PETs.

$\mathrm{H}_{30,0}$ : Oth.Users show no difference in perceptions of use between Adv.PETs and Oth.PETs.

$\mathrm{H}_{3 \mathrm{a}, 1}$ : Adv.Users show a significant difference in perceptions of use between Adv.PETs and Oth.PETs.

$\mathrm{H}_{30}, 1$ : Oth.Users show a significant difference in perceptions of use between Adv.PETs and Oth.PETs.

We also investigate other factors such as competency, as an antecedent to technology acceptance, as well as factors contributing to the use of privacy technology, such as awareness and social influence. Social influence is a factor well investigated in relation to technology adoption and usage [96, 101].

We investigate as RQ4, "Does perceived competency at managing one's privacy online differ between Adv.Users and Oth.Users?"
$\mathrm{H}_{4,0}$ : There is no difference in perceived privacy competency between Adv.Users and Oth.Users.

$\mathrm{H}_{4,1}$ : There is a significant difference in perceived privacy competency between Adv.Users and Oth.Users.

We investigate as RQ5, "Do Adv.Users' and Oth.Users' [awareneness of/perceived social influence to use] differ between Adv.PETs and Oth.PETs?" via the hypotheses

$\mathrm{H}_{5 \mathrm{a}, 0}$ : Adv.Users show no difference in [awareneness of/perceived social influence to use] between Adv.PETs and Oth.PETs.

$\mathrm{H}_{5 \mathrm{o}}, 0$ : Oth.Users show no difference in [awareneness of/perceived social influence to use] between Adv.PETs and Oth.PETs.

$\mathrm{H}_{5 \mathrm{a}, 1}$ : Adv.Users show a significant difference in awareneness of/perceived social influence to use] between Adv.PETs and Oth.PETs. $\mathrm{H}_{5 \mathrm{o}}, 1$ : Oth.Users show a significant difference in awareneness of/ perceived social influence to use] between Adv.PETs and Oth.PETs.

We further query participants on why they chose Adv.PETs or Oth.PETs, as well as the support they would need to use the other type of PETs, as RQ6.

\subsection{Method}

4.2.1 Participants. For the third study, we recruited an $N=183$ from the US, UK and DE via Prolific Academic. The demographics across the 3 countries is provided in Table 9.

4.2.2 Procedure. Study 3 consisted of the following questionnaires: (a) demographics, (b) perceived competency in protecting privacy online, (c) description of two lists of PETs (List A, the Adv.PETs and List B, the Oth.PETs) and questions on which set participants 
Table 9: Participant Characteristics

\begin{tabular}{rcrrrr}
\hline & Country & $N$ & Mean Age & \multicolumn{2}{c}{ Gender } \\
\cline { 3 - 6 } & & & & \#Female & \#Male \\
\hline \multirow{3}{*}{ Study 3} & US & 40 & 31.50 & 25 & 15 \\
& UK & 101 & 37.23 & 58 & 43 \\
& DE & 42 & 28.98 & 16 & 26 \\
\hline
\end{tabular}

\begin{tabular}{|c|c|c|}
\hline Pre-Questions & PETs List Choice & Perception of Use \\
\hline & Adv. vs Oth. PETs & Oth. PETs \\
\hline Demographics & \begin{tabular}{|c|} 
Description \& Choice b/w \\
List A (Adv.PETS \& \\
List B (Oth.PETs) \\
\end{tabular} & \multirow{3}{*}{\begin{tabular}{|l} 
Awareness \\
Perceived Usefulness \\
Perceived Ease of Use \\
Behavior Intention \\
Social Influence
\end{tabular}} \\
\hline$\nabla$ & $V$ & \\
\hline $\begin{array}{c}\text { Perceived } \\
\text { Competency } \\
\text { to protect privacy }\end{array}$ & $\begin{array}{l}\text { Open-Ended } \\
\text { Reasoning for } \\
\text { choice \& support } \\
\text { needed }\end{array}$ & \\
\hline
\end{tabular}

Figure 6: Study 3 design.

mostly use, as well as support they believe they would need to use the other set, and (d) awareness, perceived usefulness, ease of use, social influence, behavior intention to compare between the two sets of PETs. We depict the procedure in Figure 6.

4.2.3 Measurement Apparatus. This section describes the questions and scales employed in Study 3, where we employed good practice guidelines of security and privacy user studies $[18,19]$, in adapting existing scales, as much as possible.

Choice of PETs, Reasoning \& Support: We provided participants with two lists of PETs and asked "Which of the two lists contains the privacy methods that you most often use to protect your privacy online?" We then asked them to explain why they 'most often' use PETs from one list and why they 'very rarely or not at all' use methods from the other list. We further queried about what would support them to use methods from the list they did not select, in particular, what would help or encourage them. We provide the full questions and wording in the Appendix D.

Perceived Competency: We adapt Williams \& Deci's [106, 107] perceived competence questionnaire to privacy protection online. We provide the 4 items of the questionnaire in Table 14 of the Appendix E. The questionnaire is provided with a 7-point Likert scale ranging from 1 - "Not true at all" to 7 - "Very True" and a midpoint at 4 with "Somewhat True".

Technology acceptance model components: We adapt Davis [24, 25] scale for measuring the components of TAM, namely for perceived usefulness, perceived ease of use and intention to use privacy technology, as provided in Table 14 of the Appendix E. The scales were provided with 5-point Likert ranging from 1 - "Strongly Disagree" to 5 - "Strongly Agree".

Awareness \& Social Influence: We created a 4-item questionnaire to gauge awareness of PETs, as shown in Table 14 of the Appen$\operatorname{dix} \mathrm{E}$. We further adapt the social influence scale from Venkatesh et al. [102], where social influence is defined as the degree to which an individual perceives that important others believe he should use the new system. These scales are provided on a 5-point Likert ranging from 1 - "Strongly Disagree" to 5 - "Strongly Agree".
4.2.4 Limitations. Self-Report Questionnaires: Similar to Studies 1 and 2, Study 3's data collection is based on self-reports. However, in Study 3, we employ standard questionnaires with scales composed of multiple items. These scales have been widely used and validated in past research, as referenced in Section 4.2.3. We provide the assessment of internal consistency obtained in Study 3 for each scale in Table 14 of the Appendix.

Additional measures and ordering: While we carefully selected questionnaires for the purpose of Study 3, additional ones such as to elicit participants' tech-savviness could provide means of verifying the self-reported Perceived Privacy Competency questionnaire, for example. In addition, placing the demographics questionnaire at the fore may consume participants' attention span. We included attention checks throughout the study. However, in the future, we would comsider to position the demographics questionnaire at the end of the study.

\subsection{Quantitative Results}

In Study 3, we asked participants to select the list containing the PETs they most often use. We detailed the contents of the lists in Section 4.1. Only 17 of 183 participants mostly use Adv.PETs, that is the list pertaining to the least used cluster. All the remaining participants chose the Oth.PETs list. We distinguish these 2 groups of users as Adv.Users and Oth.Users.

We compute the internal consistency of the items for each of the perception scales. We provide the Cronbach $\alpha$ values in Table 14 of the Appendix E.

We investigate RQ3, How does Adv.Users' and Oth.Users' perceptions of use differ between Adv.PETs and Oth.PETs?, and RQ5, do Adv.Users' and Oth.Users' [awareneness of/perceived social influence to use] differ between Adv.PETs and Oth.PETs?

We compute pairwise $t$-tests for Adv.PET and Oth.PETs across participant responses for awareness of PETs, perceived usefulness, perceived ease of use, behavior intention and social influence. We summarise the results in Table 10, where Oth.Users showed significantly higher perceptions of Oth.PETs than of Adv.PETs, across all scales with $p<.001$. We reject the null hypotheses, $\mathrm{H}_{30}, 0$ and $\mathrm{H}_{50}, 0$ that Oth.Users show no difference in perceptions of use between Adv.PETs and Oth.PETs, for the five use perceptions shown in Table 10. Adv.Users only reported higher perceived usefulness of Adv.PETs than of Oth.PETs. We accept the null hypotheses $\mathrm{H}_{3 \mathrm{a}}, 0$ and $\mathrm{H}_{5 \mathrm{a}, 0}$ for Adv.User for all user perceptions, except for perceived usefulness.

We investigate RQ4, Does perceived competency at managing one's privacy online differ between Adv.Users and Oth.Users? We compute differences in perceived competence between Adv.Users and Oth.Users, with an independent samples Mann-Whitney U test. We do not observe a statistical significant difference in perceived competency in ensuring privacy protection online between Adv.Users and Oth.Users. We therefore accept the null hypothesis $\mathrm{H}_{4,0}$ that there is no difference in perceived privacy competency between Adv.Users and Oth.Users.

\subsection{Qualitative Results}

We investigate RQ6, that is "why do individuals choose Adv.PETs or Oth.PETs? what support would they need to use the other type 
Table 10: Within-group pairwise comparison of technology use perception of Adv.PETs vs. Oth.PETs, where Oth.Users have higher perceptions of Oth.PETs than Adv.PETs

\begin{tabular}{llllllll}
\hline \multirow{2}{*}{ Use Perception } & \multicolumn{3}{c}{ Adv.Users } & & \multicolumn{3}{c}{ Oth.Users } \\
\cline { 2 - 3 } \cline { 6 - 7 } & \multicolumn{3}{c}{$t(16)$} & $p$ & & & \multicolumn{3}{c}{$t(165)$} & $p$ \\
\hline Awareness & & & & & Oth.PETs & -16.290 & .000 \\
Perceived Usefulness & \multirow{2}{*}{ Adv.PETs } & 2.787 & .013 & & Oth.PETs & -6.999 & .000 \\
Perceived Ease of Use & & & & & Oth.PETs & -18.494 & .000 \\
Behavior Intention & & & & & Oth.PETs & -15.189 & .000 \\
Social Influence & & & & & Oth.PETs & -9.231 & .000 \\
\hline
\end{tabular}

of PETs?" This section describes the coding process and reports responses for (1) users of both types of PETs, (2) users of Adv.PETs and (3) users of Oth.PETs. Note that List A in the survey refers to Adv.PETs and List B refers to Oth.PETs.

4.4.1 Codebook Creation. The sample of $N=183$ participants in Study 3 were asked two open free-form questions: Q1, why they mostly use methods from one list, and Q2, what would support them to use methods from the other list. Responses were required to be at least 30 words long, with no maximum.

We facilitated a conventional line by line coding, where $n=50$ responses were coded by one coder to extract concepts from the free-form text. This process has been used in usable privacy research before and is well accepted [20]. The concepts were grouped into 5 categories for each question, and the coding was validated and refined with discussions with another coder. We used the set of categories and concepts to create a codebook. We trained 2 researchers as coders and further refined the codebook with a final set of 47 codes within 5 categories as provided in Table 15 of the Appendix F. The '-other' codes, such as EFF03-other were added to include concepts not initially catered for in the codebook.

We evaluate inter-rater reliability via \%-agreement and Cohen $k$ [43, 67] on 100 responses across the 47 codes. We find that the coders were on agreement $96 \%$ of the time and there was a substantial agreement with Cohen $\mathrm{k}$ of .837, $p<.001$.

4.4.2 Use of both types of PETs. We note that 35 of the 183 participants reported using PETs from both lists, where most of them reported using Oth.PETs primarily and a few Adv.PETs. The few Adv.PETs that participants reported using are VPN (mentioned by $n=14$ ), NoScript, DuckDuckGo, Proxy, anti-tracking, Kaspersky and Ghostery. Note that as described in Section 4.2.2, List A refers to Adv.PET whereas List B refers to Oth.PETs.

Participants explained that they use Oth.PETs as baseline and Adv.PETs for critical conditions or as a form of layered protection, such as expressed by P121 in 'I think methods on List B are things that should use [sic] everybody, the bare minimum you should use while surfing on the world wide web. I also use DuckDuckGo, VPN, NoScript and Ghostery, but the things on the other List are more often', P9 in ' $i$ [sic] do use some from List A, however list B come pretty standard with my tech as well as is free and easy to use.' and $\mathrm{P} 127$ in 'I mostly use adblock on very restrictive settings, noscript, no location tracking and private browsing, because these methods are the most convenient ones. I sometimes use VPN and proxies as well, but since this requires more action, I only use this in critical cases.'
In addition, 2 participants reported using VPN for other reasons than for privacy protection, with for example P112 stating 'clear cookies and clear the history is a thing $i$ [sic] do every day [...] ok sometimes $i$ [sic] use VPN, but not to protect myself only to watch movies from the US, which are geo blocked' and P25 'I will occasionally use a VPN if I need to access websites that are not available without.'

4.4.3 Users of Advanced PETs. Users of Adv.PETs reasoned that Adv.PETs are more effective in ensuring their privacy online, such as reported by P117 in 'List A contains tried and tested methods of protecting privacy, list B is superficial' or P105 in 'list B not effective'. They also pointed to the trustworthiness of Adv.PETs, as expressed by $\mathrm{P} 110$ 'Tor is more trustworthy' or P40 'List A is [sic] better to rely on'.

4.4.4 Users of Other PETs. Overall we find that participants' reasons for mostly using Oth.PETs corroborated with the support and encouragement they believe they would need to use Adv.PETs, as similar themes emerged from the two open-ended questions. We consequently provide the responses for the two open-ended questions based on the themes. From the 5 categories of Table 15, we observe recurrent themes in the responses, namely (1) 'information about' the PETs, (2) 'usability' type responses, (3) level of 'privacy or protection needed', (4) 'cost' of PET, (5) 'trust and reliability' of PET and (6) 'social support' to use PET. We report participants' reasoning for choosing Oth.PETs and support needed to use Adv.PETs across these themes in Table 11.

\section{DISCUSSION}

We organise this section into four main sections, with each providing a brief summary of findings, followed with a discussion of the implications of our findings as well as their relation to previous research. We also highlight lines of investigation for future research.

\subsection{Usage Pattern of Privacy Methods}

Summary of findings: We visualised privacy methods use via a cluster map, and relations between methods and country of use via a spatial map. We identified three clusters pertaining to three distinct privacy method use patterns, with the left and right clusters respectively showing the least and most used methods.

Interpretation of Clusters. A visual inspection of the clusters reveals insights into participants' privacy methods preference, such as, that the right cluster contains simple, easy to use, and convenient methods of protection, that can be said to be more inclusive of skill levels and are more mainstream, while the leftmost cluster contains more advanced PETs.

Use of the classification. The results of the clustering may be used to support users with recommendations, such as suggesting other privacy methods in the same cluster, with the knowledge that users with similar methods preference used these other methods, and the underlying assumption that methods in the same cluster share similarity in ease of use and perceived required skill. The classification is also in itself a methodological tool, that can facilitate further user-centric investigation of PETs. 
Table 11: Qualitative Responses from n=166 participants who chose Oth.PETs: reason for choice $\&$ support/encouragement needed to use Adv.PETs

\begin{tabular}{|c|c|c|c|c|c|}
\hline & \multicolumn{2}{|l|}{ Theme 1: Information About } & \multicolumn{3}{|c|}{ Theme 2: Usability } \\
\hline & & $\%$ & & & $\%$ \\
\hline \multirow[t]{8}{*}{ Reasons } & Not know Adv.PETs & 28.92 & Reasons & Easy to use/install Oth.PETs & 24.10 \\
\hline & Familiar with Oth.PETs & 27.11 & & Adv.PETs complicated to use/install & 7.83 \\
\hline & Technical skills required & 6.02 & & Oth.PETs are readily available & 6.63 \\
\hline & Belief Oth.PETs are for casual/non-tech savvy users & $<4$ & & Oth.PETs easy to access & 5.42 \\
\hline & Need to be advised/recommended & $<4$ & & Oth.PETs convenient to use & 4.82 \\
\hline & & & & Amount of work vs benefit gained from Adv.PETs & 4.82 \\
\hline & & & & Oth.PETs integrated in service & $<4$ \\
\hline & & & & Annoyance or discomfort when using Adv,PETs & $<4$ \\
\hline \multirow[t]{5}{*}{ Support } & What they are/do & 36.14 & Support & Ease of use & 14.46 \\
\hline & How to use, training & 16.27 & & Ease of installation and configuration & 12.05 \\
\hline & Via info channels e.g. SNS, tutorials, trusting list & 9.04 & & & \\
\hline & Theme 3: Privacy \& Protection Needed & & & Theme 4: Cost & \\
\hline & & $\%$ & & & $\%$ \\
\hline \multirow[t]{6}{*}{ Reasons } & For protection provided ('keeps me private') & 15.06 & Reasons & Having to pay for/cost of Adv.PETS & 14.46 \\
\hline & Oth.PETs good enough for privacy needed & 10.84 & & Oth.PETs are free & 4.22 \\
\hline & Successful experience in using Oth.PETs & 6.63 & & & \\
\hline & No need for privacy, nothing to hide & 5.42 & & & \\
\hline & Adv.PETs would be extreme/Oth.PETs for everyday use & 4.22 & & & \\
\hline & Privacy vs online experience & $<4$ & & & \\
\hline \multirow[t]{5}{*}{ Support } & If need specific PET protection functionality (e.g. anonymity) & 9.04 & Support & If Adv.PETs were free/affordable & 16.87 \\
\hline & If Adv.PETs provided more privacy than what they use & 8.43 & & & \\
\hline & If they had a bigger privacy need & 6.02 & & & \\
\hline & Theme 5: Trust \& Reliability & & & Theme 6: Social Support & \\
\hline & & $\%$ & & & $\%$ \\
\hline Reasons & Trust in PETs & 9.04 & Reasons & $\mathrm{n} / \mathrm{a}$ & \\
\hline \multirow[t]{3}{*}{ Support } & If Adv.PETs did not look fake/phoney & 5.42 & Support & Recommended by reputable company & 5.42 \\
\hline & & & & If someone they know/trust used it & $<4$ \\
\hline & & & & If someone taught them how to use or install & $<4$ \\
\hline
\end{tabular}

Cluster Transitions. We consider questions for future research into facilitating a transition from using a right-cluster method to a left-cluster method, such as 'under what conditions do users transition right to left? what is the influence of more privacy concern, more skill, a realisation that simple PETs are not enough or the influence by social contacts?' 'how to support users to shift from right to left or consolidate his position in the current cluster?' 'how can the methods in the different clusters be used in a layered approach for more complete protection?'

Cross-National Visualisation. The spatial plot of privacy methods in relation to country (of Figure 5) clearly shows a gravitation of German participants towards more advanced and active privacy methods, compared to US and UK participants. This may be a reflection of Germans' privacy perceptions, where previous research reported that Germans, compared to participants of other countries, found controlling access to personal data, private realms and data protection more important [94], were among those most sensitive to the duration and quantity of data collection (location data) [22], or attributed a higher probability to privacy violations on SNS [56]. Figure 5 may also support discussions and further investigations of the effectiveness of privacy campaigns, of the role of the media or social connections in a particular country.

\subsection{Non-Technology Methods in Top 10}

Summary of findings: Among the similarities in privacy methods usage across the three countries (from Study 2, Table 8), we find that non-technology methods (1) of being careful of websites, (2) to limit sharing, (3) research before engaging (2 out of 3 countries), (4) not subscribe to newsletters, and (5) not access accounts in public places, appear in the most used methods across countries.

For the three countries, these non-technology methods made up 4 or 5 of the top 10 most preferred privacy methods thereby demonstrating that users rely more on their own means to protect themselves than privacy technologies. This raises questions about the reasons for reliance on non-technology methods rather than PETs, such as 'are users concerned enough and aware of PETs to use them? how were their previous experience with PETs?'

\subsection{Perception of Use}

Summary of findings: $9 \%$ of participants in Study 3 reported to use Adv.PETs, $91 \%$ to use Oth.PETs and $19 \%$ to use PETs from both lists. The low preference for Adv.PETs corresponds to previous research findings of no mention or of low use of Adv.PETs [39, 75].

Study 3 enabled us to evaluate perception of use of PETs within different clusters. From Table 10, it is clear that Oth.Users have higher use perceptions of Oth.PETs than Adv.PETs, which may highlight a perceptive barrier to individuals' use of Adv.PETs. Similar to previous research of the importance of perceived usefulness for the adoption of anonymous credentials [12, 46], our respondents who reported to use Adv.PETs showed higher perceived usefulness of Adv.PETs than Oth.PETs.

In addition. users of Adv.PETs and Oth.PETs did not report a difference in their perceived competency in protecting their privacy online. While perceived competence may predict continuance or 
persistence in behavior [73, 88], given that individuals may feel more motivated to perform a task when they feel competent in, it may be complex to change individuals' behavior patterns from habitual use of Oth.PETs.

\subsection{PETs Choice and Support Needed}

Summary of findings. The reported responses of Oth.Users in Study 3 support and extend the findings of previous research on factors influencing the active use of PETs (summarised earlier in Table 1 of Section 2.1.2). We report Oth.Users' responses in Table 11 and discuss them below.

Our findings contribute to previous research with a collective perspective that is neither specific to particular PETs nor contextual to use scenario, such as social network or e-commerce. Our methodology may therefore help to identify general beliefs and biases of users that act as obstacles to the use of Adv.PETs. In particular, Table 11 depicts a picture of 'don't know, therefore don't use Adv.PETs', and yet have perceptions that Adv.PETs are not easy to use/install, are costly, or are not trustworthy. Therefore, further to being aware of Adv.PETs, individuals may also have to overcome the biased perceptions they hold about these PETs.

Awareness of PETs (from Theme 1: Information about). Our participants reported to not knowing about Adv.PETs and would benefit with an understanding of what they are and what they do. This is similar to previous reports on awareness of protection tools or inability to use protection for tracking or end-to-end encryption [87, 93]. In addition, we also found beliefs of technical skills requirement, and requiring training or information via social media and other channels.

Usability (Theme 2). Our participants also expressed a belief that Adv.PETs are not easy to use or to install, with other usabilityrelated beliefs listed in Table 11 . Ease of use for anonymity technology [12, 46] and usability for secure communication [1,87] were also reported in previous research. However, although usability was previously found to not be more important than other factors (such as risk of sharing in social network [36], contextual aspects of messaging tool [1] or perceived usefulness [12]), for our participants, one of the most important reason for using Oth.PETs, rather than Adv.PETs, was ease of use and configuration, which may be a biased perception about Adv.PETs' usability.

Usefulness (Theme 3: Privacy \& Protection Needed). Our participants reported to use Oth.PETs for the protection provided, that Oth.PETs are good enough, that they had a successful use experience with Oth.PETs, and that if they were to use Adv.PETs, it would be for other specific protection functionalities that are not provided by Oth.PETs. While this points to their satisfaction in using Oth.PETs, being able to compare the privacy protection provided between Oth.PETs and Adv.PETs, may provide users with a wider choice.

Need for Privacy (Theme 3: Privacy \& Protection Needed). Some of our participants reported to not needing privacy/have nothing to hide, or that using Adv.PETs would be extreme. These loosely match previous reports of no perceived need to act for tracking protection and end-to-end encryption technologies [87, 93]. This depicts how ill-informed users may be with regards to their privacy online, in particular not knowing how much more protection Adv.PETs can offer them, as well as not realising the potential extent of privacy loss and online harms in the data-centric web.

Cost (Theme 4). Our participants referenced the perceived monetary cost Adv.PETs to be a deterrent to their use. In comparison, previous research investigations in relation to cost included the perceived value of information [22], monetary reward for disclosure [45], cost and benefits tradeoffs of disclosure [29], cost of privacy breaches [2] or the time spent reading privacy policies [66].

Trust \& Social Support (Themes 5 \& 6). PETs' trustworthiness and recommendation (either socially or accredited via a reputable company), were also deemed important, as well as social support to recommend or teach how to use Adv.PETs. Trust has also been considered before in investigations involving the active use of privacy controls [36] and adoption of PETs [12, 46], and in relation to accessible and salient privacy information in websites [99], while social influence had links with the use of privacy [1,39] and security [23] controls. While neutral accredited lists already exist via privacy-promoting organisations such as the Electronic Frontier Foundation, the influence of social connections and support from those individuals know and/or trust may have a 'multiplier' effect in increasing use of Adv.PETs. Social influence and support can facilitate integration of PETs into daily life, expand use to more casual users, as well as help sustain use.

\section{CONCLUSION}

This paper provides a large scale, cross-national investigation of the use of a collection of privacy methods online, derives patterns of use and follows-up with an investigation of perception of use of PETs between the patterns. The usage classification, while contributing to our knowledge of PETs use, also provides a methodological contribution to the field. It can further be used in future user-centric investigations of PETs.

The clusters raise a number of questions about both their components and the conditions for transitioning to the least popular cluster. In addition, by investigating individuals' own perception of use of PETs as well as the support they believe they would need to use more advanced PETs, we highlighted a few themes that may help to expand the use of advanced PETs. We recommend investigations of the questions raised in the discussion section, as future work, as well as for designers to consider the reasoning and support needed by individuals to use PETs (in particular, more advanced PETs), as provided in this paper.

\section{ACKNOWLEDGMENTS}

This research was supported by a Newcastle University Research Fellowship (Academic Track). I am thankful for the feedback provided by colleagues, all from Newcastle University, namely, Prof. Aad van Moorsel, Dr. Jaume Bacardit, Dr. Changyu Dong and Dr. Magdalene Ng. I am also grateful for the feedback and comments provided by the anonymous reviewers of CCS'20 .

\section{REFERENCES}

[1] Ruba Abu-Salma, M Angela Sasse, Joseph Bonneau, Anastasia Danilova, Alena Naiakshina, and Matthew Smith. 2017. Obstacles to the adoption of secure communication tools. In 2017 IEEE Symposium on Security and Privacy (SP). IEEE, 137-153.

[2] Alessandro Acquisti, Allan Friedman, and Rahul Telang. 2006. Is there a cost to privacy breaches? An event study. ICIS 2006 Proceedings (2006), 94. 
[3] Alessandro Acquisti and Ralph Gross. 2006. Imagined communities: Awareness, information sharing, and privacy on the Facebook. In Privacy enhancing technologies. Springer, 36-58.

[4] Alessandro Acquisti and Jens Grossklags. 2005. Privacy and rationality in individual decision making. IEEE Security \& Privacy 2 (2005), 24-30.

[5] Brooke Auxier, Lee Rainie, Monica Anderson, Andrew Perrin, Madhu Kumar, and Erica Turner. 2019. Americans and privacy: Concerned, confused and feeling lack of control over their personal information. Pew Research Center: Internet, Science \& Tech (blog). November 15 (2019), 2019.

[6] Kallol Bagchi, Robert Cerveny, Paul Hart, and Mark Peterson. 2003. The influence of national culture in information technology product adoption. AMCIS 2003 Proceedings (2003), 119.

[7] VenuGopal Balijepally, George Mangalaraj, and Kishen Iyengar. 2011. Are we wielding this hammer correctly? A reflective review of the application of cluster analysis in information systems research. Fournal of the Association for Information Systems 12, 5 (2011), 1.

[8] Susan B Barnes. 2006. A privacy paradox: Social networking in the United States. First Monday 11, 9 (2006).

[9] Susanne Barth and Menno DT De Jong. 2017. The privacy paradox-Investigating discrepancies between expressed privacy concerns and actual online behavior- $A$ systematic literature review. Telematics and informatics 34, 7 (2017), 1038-1058.

[10] Steven Bellman, Eric J Johnson, Stephen J Kobrin, and Gerald L Lohse. 2004. International differences in information privacy concerns: A global survey of consumers. The Information Society 20, 5 (2004), 313-324.

[11] Mike Bendixen. 1996. A practical guide to the use of correspondence analysis in marketing research. Marketing Research On-Line 1, 1 (1996), 16-36.

[12] Zinaida Benenson, Anna Girard, and Ioannis Krontiris. 2015. User Acceptance Factors for Anonymous Credentials: An Empirical Investigation.. In WEIS.

[13] Grant Blank, William H Dutton, and Julia Lefkowitz. 2019. Perceived Threats to Privacy Online: The Internet in Britain, the Oxford Internet Survey, 2019. (2019).

[14] Badrish Chandramouli, Jonathan Goldstein, Xin Jin, Balan Sethu Raman, and Songyun Duan. 2013. Real-time-ready behavioral targeting in a large-scale advertisement system. US Patent 8,442,863.

[15] Ramnath K Chellappa and Raymond G Sin. 2005. Personalization versus privacy: An empirical examination of the online consumer's dilemma. Information Technology and Management 6, 2-3 (2005), 181-202.

[16] Hichang Cho, Milagros Rivera-Sánchez, and Sun Sun Lim. 2009. A multinational study on online privacy: global concerns and local responses. New media \& society 11, 3 (2009), 395-416.

[17] Lizzie Coles-Kemp and Elahe Kani-Zabihi. 2011. Practice makes perfect: motivating confident privacy protection practices. In 2011 IEEE Third International Conference on Privacy, Security, Risk and Trust and 2011 IEEE Third International Conference on Social Computing. IEEE, 866-871.

[18] Kovila P.L. Coopamootoo and Thomas Groß. 2017. A Codebook for Evidence Based Research: The Nifty Nine Completeness Indicators v1.1. Technical Report 1514. Newcastle University.

[19] Kovila PL Coopamootoo and Thomas Groß. 2017. Cyber Security and Privacy Experiments: A Design and Reporting Toolkit. In IFIP International Summer School on Privacy and Identity Management. Springer, 243-262.

[20] Kovila PL Coopamootoo and Thomas Groß. 2017. Why Privacy is All But Forgotten - An Empirical Study of Privacy and Sharing Attitude. Proceedings on Privacy Enhancing Technologies 4 (2017), 39-60.

[21] Lynne Coventry, Debora Jeske, and Pamela Briggs. 2014. Perceptions and actions: Combining privacy and risk perceptions to better understand user behaviour. (2014).

[22] Dan Cvrcek, Marek Kumpost, Vashek Matyas, and George Danezis. 2006. A study on the value of location privacy. In Proceedings of the 5th ACM workshop on Privacy in electronic society. 109-118.

[23] Sauvik Das, Adam DI Kramer, Laura A Dabbish, and Jason I Hong. 2015. The role of social influence in security feature adoption. In Proceedings of the 18th ACM conference on computer supported cooperative work \& social computing. 1416-1426.

[24] Fred D Davis. 1989. Perceived usefulness, perceived ease of use, and user acceptance of information technology. MIS quarterly (1989), 319-340.

[25] Fred D Davis, Richard P Bagozzi, and Paul R Warshaw. 1989. User acceptance of computer technology: a comparison of two theoretical models. Management science 35, 8 (1989), 982-1003.

[26] Tobias Dienlin and Sabine Trepte. 2015. Is the privacy paradox a relic of the past? An in-depth analysis of privacy attitudes and privacy behaviors. European Journal of Social Psychology 45, 3 (2015), 285-297.

[27] Tamara Dinev, Valentina Albano, Heng Xu, Alessandro D'Atri, and Paul Hart. 2016. Individuals' attitudes towards electronic health records: A privacy calculus perspective. In Advances in healthcare informatics and analytics. Springer, 19-50.

[28] Tamara Dinev, Massimo Bellotto, Paul Hart, Vincenzo Russo, Ilaria Serra, and Christian Colautti. 2006. Privacy calculus model in e-commerce-a study of Italy and the United States. European fournal of Information Systems 15, 4 (2006) 389-402.
[29] Tamara Dinev and Paul Hart. 2006. An extended privacy calculus model for e-commerce transactions. Information systems research 17, 1 (2006), 61-80.

[30] Daniel Ebbert and Stephan Dutke. 2020. Patterns in students' usage of lecture recordings: a cluster analysis of self-report data. Research in Learning Technology 28 (2020).

[31] Benjamin G Edelman and Michael Luca. 2014. Digital discrimination: The case of Airbnb. com. Harvard Business School NOM Unit Working Paper 14-054 (2014).

[32] ENISA. 2020. Privacy Enhancing Technologies. https://www.enisa.europa.eu/ topics/data-protection/privacy-enhancing-technologies

[33] Abdul Azeez Erumban and Simon B De Jong. 2006. Cross-country differences in ICT adoption: A consequence of Culture? Journal of world business 41, 4 (2006), 302-314.

[34] Special Eurobarometer. 2019. The General Data Protection Regulation - Special Eurobarometer 487a. Special Eurobarometer (2019).

[35] Forbes-Insights. 2019. Rethinking Privacy in the AI Era. https://www.forbes. com/sites/insights-intelai/2019/03/27/rethinking-privacy-for-the-ai-era/

[36] Vaibhav Garg, Kevin Benton, and L Jean Camp. 2014. The privacy paradox: a Facebook case study. In 2014 TPRC conference paper.

[37] R Kelly Garrett. 2019. Social media's contribution to political misperceptions in US Presidential elections. PloS one 14, 3 (2019), e 0213500.

[38] Nina Gerber, Paul Gerber, and Melanie Volkamer. 2018. Explaining the privacy paradox: A systematic review of literature investigating privacy attitude and behavior. Computers \& Security 77 (2018), 226-261.

[39] Nina Gerber, Verena Zimmermann, and Melanie Volkamer. 2019. Why Johnny Fails to Protect his Privacy. In 2019 IEEE European Symposium on Security and Privacy Workshops (EuroS\&PW). IEEE, 109-118.

[40] Michael Greenacre. 2017. Correspondence analysis in practice. Chapman and Hall/CRC.

[41] Michael J Greenacre. 1988. Clustering the rows and columns of a contingency table. Fournal of Classification 5, 1 (1988), 39-51.

[42] Joseph F Hair, William C Black, Barry J Babin, Rolph E Anderson, Ronald L Tatham, et al. 1998. Multivariate data analysis. Vol. 5. Prentice hall Upper Saddle River, NJ.

[43] Kevin A Hallgren. 2012. Computing inter-rater reliability for observational data: an overview and tutorial. Tutorials in quantitative methods for psychology 8, 1 (2012), 23

[44] Dara Hallinan, Michael Friedewald, and Paul McCarthy. 2012. Citizens' perceptions of data protection and privacy in Europe. Computer law \& security review 28, 3 (2012), 263-272.

[45] Il-Horn Hann, Kai-Lung Hui, Tom Lee, and I Png. 2002. Online information privacy: Measuring the cost-benefit trade-off. ICIS 2002 proceedings (2002), 1.

[46] David Harborth and Sebastian Pape. 2018. Examining technology use factors of privacy-enhancing technologies: the role of perceived anonymity and trust. (2018).

[47] Eszter Hargittai et al. 2010. Facebook privacy settings: Who cares? First Monday (2010).

[48] Geert Hofstede. 1984. Culture's consequences: International differences in workrelated values. Vol. 5. sage.

[49] Geert Hofstede. 2001. Culture's consequences: Comparing values, behaviors, institutions and organizations across nations. Sage publications.

[50] Hsiao-Ying Huang and Masooda Bashir. 2016. Privacy by region: Evaluation online users' privacy perceptions by geographical region. In 2016 Future Technologies Conference (FTC). IEEE, 968-977.

[51] Index-Mundi. 2019. Germany Demographics Profile 2019. https://www. indexmundi.com/germany/demographics_profile.html

[52] Alboukadel Kassambara and Fabian Mundt. 2017. Package 'factoextra'. Extract and visualize the results of multivariate data analyses 76 (2017).

[53] Leonard Kaufman and Peter J Rousseeuw. 2009. Finding groups in data: an introduction to cluster analysis. Vol. 344. John Wiley \& Sons.

[54] Flavius Kehr, Tobias Kowatsch, Daniel Wentzel, and Elgar Fleisch. 2015. Blissfully ignorant: the effects of general privacy concerns, general institutional trust, and affect in the privacy calculus. Information Systems fournal 25, 6 (2015), 607-635.

[55] Spyros Kokolakis. 2017. Privacy attitudes and privacy behaviour: A review of current research on the privacy paradox phenomenon. Computers \& Security 64 (2017), 122-134.

[56] Hanna Krasnova and Natasha F Veltri. 2010. Privacy calculus on social networking sites: Explorative evidence from Germany and USA. In 2010 43rd Hawaii international conference on system sciences. IEEE, 1-10.

[57] Muniruddeen Lallmahamood. 1970. An Examination of IndividualÃ Â Â s Perceived Security and Privacy of the Internet in Malaysia and the Influence of This on Their Intention to Use E-Commerce: Using An Extension of the Technology Acceptance Model. The fournal of Internet Banking and Commerce 12, 3 (1970), 1-26.

[58] Phillippa Lally, Cornelia HM Van Jaarsveld, Henry WW Potts, and Jane Wardle. 2010. How are habits formed: Modelling habit formation in the real world. European journal of social psychology 40, 6 (2010), 998-1009.

[59] Nancy K Lankton, D Harrison McKnight, and John F Tripp. 2017. Facebook privacy management strategies: A cluster analysis of user privacy behaviors. 
Computers in Human Behavior 76 (2017), 149-163.

[60] Paul Legris, John Ingham, and Pierre Collerette. 2003. Why do people use information technology? A critical review of the technology acceptance model Information \& management 40, 3 (2003), 191-204.

[61] David John Lemay, Tenzin Doleck, and Paul Bazelais. 2017. "Passion and concern for privacy" as factors affecting snapchat use: A situated perspective on technology acceptance. Computers in Human Behavior 75 (2017), 264-271.

[62] Yanchi Liu, Zhongmou Li, Hui Xiong, Xuedong Gao, and Junjie Wu. 2010. Understanding of internal clustering validation measures. In 2010 IEEE International Conference on Data Mining. IEEE, 911-916.

[63] James MacQueen et al. 1967. Some methods for classification and analysis of multivariate observations. In Proceedings of the fifth Berkeley symposium on mathematical statistics and probability, Vol. 1. Oakland, CA, USA, 281-297.

[64] Mary Madden, Lee Rainie, Kathryn Zickuhr, Maeve Duggan, and Aaron Smith 2014. Public perceptions of privacy and security in the post-Snowden era. Pew Research Center 12 (2014)

[65] Bryan A Marshall, Peter W Cardon, Daniel T Norris, Natalya Goreva, and Ryan D'Souza. 2008. Social networking websites in India and the United States: A cross-national comparison of online privacy and communication. Issues in Information Systems 9, 2 (2008), 87-94.

[66] Aleecia M McDonald and Lorrie Faith Cranor. 2008. The cost of reading privacy policies. Isjlp 4 (2008), 543.

[67] Nora McDonald, Sarita Schoenebeck, and Andrea Forte. 2019. Reliability and inter-rater reliability in qualitative research: Norms and guidelines for CSCW and HCI practice. Proceedings of the ACM on Human-Computer Interaction 3 , CSCW (2019), 1-23.

[68] Caroline Lancelot Miltgen, Aleš Popovič, and Tiago Oliveira. 2013. Determinants of end-user acceptance of biometrics: Integrating the "Big 3" of technology acceptance with privacy context. Decision Support Systems 56 (2013), 103-114.

[69] Caroline Lancelot Miltgen and H Jeff Smith. 2015. Exploring information privacy regulation, risks, trust, and behavior. Information \& Management 52, 6 (2015), 741-759.

[70] Madhumita Murgia and Max Harlow. 2019. How top health websites are sharing sensitive data with advertisers. https:/www.ft.com/content/0fbf $4 \mathrm{~d} 8 \mathrm{e}-022 \mathrm{~b}-$ 11ea-be59-e49b2a136b8d

[71] Moses Namara, Daricia Wilkinson, Kelly Caine, and Bart P Knijnenburg. 2020 Emotional and Practical Considerations Towards the Adoption and Abandonment of VPNs as a Privacy-Enhancing Technology. Proceedings on Privacy Enhancing Technologies 2020, 1 (2020), 83-102.

[72] Patricia A Norberg, Daniel R Horne, and David A Horne. 2007. The privacy paradox: Personal information disclosure intentions versus behaviors. Fournal of Consumer Affairs 41, 1 (2007), 100-126.

[73] Michael Oduor and Harri Oinas-Kukkonen. 2017. Commitment devices as behavior change support systems: a study of users' perceived competence and continuance intention. In International Conference on Persuasive Technology. Springer, 201-213.

[74] Judith S Olson, Jonathan Grudin, and Eric Horvitz. 2005. A study of preferences for sharing and privacy. In CHI'05 extended abstracts on Human factors in computing systems. 1985-1988.

[75] Isabelle Oomen and Ronald Leenes. 2008. Privacy risk perceptions and privacy protection strategies. In Policies and research in identity management. Springer 121-138.

[76] Yong Jin Park. 2015. Do men and women differ in privacy? Gendered privacy and (in) equality in the Internet. Computers in Human Behavior 50 (2015), 252-258.

[77] UK Parliament. 2018. Data Protection Act 2018. URL https://services. parliament. uk/bills/2017-19/dataprotection. html (2018).

[78] Eyal Peer, Laura Brandimarte, Sonam Samat, and Alessandro Acquisti. 2017. Beyond the Turk: Alternative platforms for crowdsourcing behavioral research Journal of Experimental Social Psychology 70 (2017), 153-163.

[79] DLA Piper. 2019. Data protection Laws of the world. 2019.

[80] Denise F Polit and Cheryl Tatano Beck. 2010. Generalization in quantitative and qualitative research: Myths and strategies. International journal of nursing studies 47, 11 (2010), 1451-1458.

[81] Sören Preibusch. 2013. Guide to measuring privacy concern: Review of survey and observational instruments. International Journal of Human-Computer Studies 71, 12 (2013), 1133-1143.

[82] Privacy-International. 2019. No Body's Business But Mine: How Menstruation Apps Are Sharing Your Data. https://privacyinternational.org/long-read/3196/ no-bodys-business-mine-how-menstruation-apps-are-sharing-your-data

[83] PWC. 2016. Data breach notification: 10 ways GDPR differs from the US model. https://www.pwc.com/us/en/services/consulting/cybersecurity/library/ broader-perspectives/gdpr-differences.html
[84] Rupak Rauniar, Greg Rawski, Jei Yang, and Ben Johnson. 2014. Technology acceptance model (TAM) and social media usage: an empirical study on Facebook. fournal of Enterprise Information Management (2014).

[85] Elissa M Redmiles, Ziyun Zhu, Sean Kross, Dhruv Kuchhal, Tudor Dumitras, and Michelle L Mazurek. 2018. Asking for a friend: Evaluating response biases in security user studies. In Proceedings of the 2018 ACM SIGSAC Conference on Computer and Communications Security. 1238-1255.

[86] Philip J Reed, Emma S Spiro, and Carter T Butts. 2016. Thumbs up for privacy?: Differences in online self-disclosure behavior across national cultures. Social science research 59 (2016), 155-170.

[87] Karen Renaud, Melanie Volkamer, and Arne Renkema-Padmos. 2014. Why doesn't Jane protect her privacy?. In International Symposium on Privacy Enhancing Technologies Symposium. Springer, 244-262.

[88] Juan Carlos Roca and Marylène Gagné. 2008. Understanding e-learning continuance intention in the workplace: A self-determination theory perspective. Computers in human behavior 24, 4 (2008), 1585-1604.

[89] Juan Carlos Roca, Juan José García, and Juan José De La Vega. 2009. The importance of perceived trust, security and privacy in online trading systems. Information Management \& Computer Security (2009).

[90] Charles Romesburg. 2004. Cluster analysis for researchers. Lulu. com.

[91] Peter J Rousseeuw. 1987. Silhouettes: a graphical aid to the interpretation and validation of cluster analysis. Journal of computational and applied mathematics 20 (1987), 53-65.

[92] Eva-Maria Schomakers, Chantal Lidynia, Dirk Müllmann, and Martina Ziefle. 2019. Internet users' perceptions of information sensitivity-insights from germany. International fournal of Information Management 46 (2019), 142-150.

[93] Fatemeh Shirazi and Melanie Volkamer. 2014. What deters Jane from preventing identification and tracking on the Web?. In Proceedings of the 13th Workshop on Privacy in the Electronic Society. 107-116.

[94] Tal Soffer and Anat Cohen. 2014. Privacy perception of adolescents in a digital world. Bulletin of Science, Technology \& Society 34, 5-6 (2014), 145-158.

[95] Sarah Spiekermann, Jens Grossklags, and Bettina Berendt. 2001. E-privacy in 2nd generation E-commerce: privacy preferences versus actual behavior. In Proceedings of the $3 r d$ ACM conference on Electronic Commerce. ACM, 38-47.

[96] Majharul Talukder and Ali Quazi. 2011. The impact of social influence on individuals' adoption of innovation. Fournal of Organizational Computing and Electronic Commerce 21, 2 (2011), 111-135.

[97] Robert Thomson, Masaki Yuki, and Naoya Ito. 2015. A socio-ecological approach to national differences in online privacy concern: The role of relational mobility and trust. Computers in Human Behavior 51 (2015), 285-292.

[98] Sigal Tifferet. 2019. Gender differences in privacy tendencies on social network sites: a meta-analysis. Computers in Human Behavior 93 (2019), 1-12.

[99] Janice Y Tsai, Serge Egelman, Lorrie Cranor, and Alessandro Acquisti. 2011. The effect of online privacy information on purchasing behavior: An experimental study. Information systems research 22, 2 (2011), 254-268.

[100] Sonja Utz and Nicole Kramer. 2009. The privacy paradox on social network sites revisited: The role of individual characteristics and group norms. Cyberpsychology: Journal of Psychosocial Research on Cyberspace 3, 2 (2009), 2.

[101] Sandra A Vannoy and Prashant Palvia. 2010. The social influence model of technology adoption. Commun. ACM 53, 6 (2010), 149-153.

[102] Viswanath Venkatesh, Michael G Morris, Gordon B Davis, and Fred D Davis. 2003. User acceptance of information technology: Toward a unified view. MIS quarterly (2003), 425-478.

[103] Leo R Vijayasarathy. 2004. Predicting consumer intentions to use on-line shopping: the case for an augmented technology acceptance model. Information \& management 41, 6 (2004), 747-762.

[104] Paul Voigt and Axel Von dem Bussche. 2017. The eu general data protection regulation (gdpr). A Practical Guide, 1st Ed., Cham: Springer International Publishing (2017).

[105] Yang Wang, Gregory Norcie, Saranga Komanduri, Alessandro Acquisti, Pedro Giovanni Leon, and Lorrie Faith Cranor. 2011. " I regretted the minute I pressed share" a qualitative study of regrets on Facebook. In Proceedings of the seventh symposium on usable privacy and security. 1-16.

[106] Geoffrey Williams, Zachary Freedman, and Edward Deci. 1998. Supporting autonomy to motivate glucose control in patients with diabetes. Diabetes $47,1 \mathrm{~S}$ (1998).

[107] Geoffrey C Williams and Edward L Deci. 1996. Internalization of biopsychosocial values by medical students: a test of self-determination theory. Fournal of personality and social psychology 70, 4 (1996), 767.

[108] Svante Wold, Kim Esbensen, and Paul Geladi. 1987. Principal component analysis. Chemometrics and intelligent laboratory systems 2, 1-3 (1987), 37-52.

[109] Jeremy Wright and Sajid Javid. 2019. HM Gov Online harms white paper. April 2019. (2019). 


\section{A STUDY 2 QUESTIONNAIRE}

The 43 privacy methods used in the questionnaire in Study 2 were named by participants themselves in Study 1 and compiled into 43 distinct methods.

The instruction was "From the list below, please rate the tools/methods in terms of whether you have used them very often before versus not used at all or very rarely, to achieve the purpose of privacy online."

Table 12: The list of methods as provided in a tabular form in the questionnaire \& listed in random order for each participant.

\begin{tabular}{|c|c|c|}
\hline Tools/Methods & very often & not used at all or very rarely \\
\hline 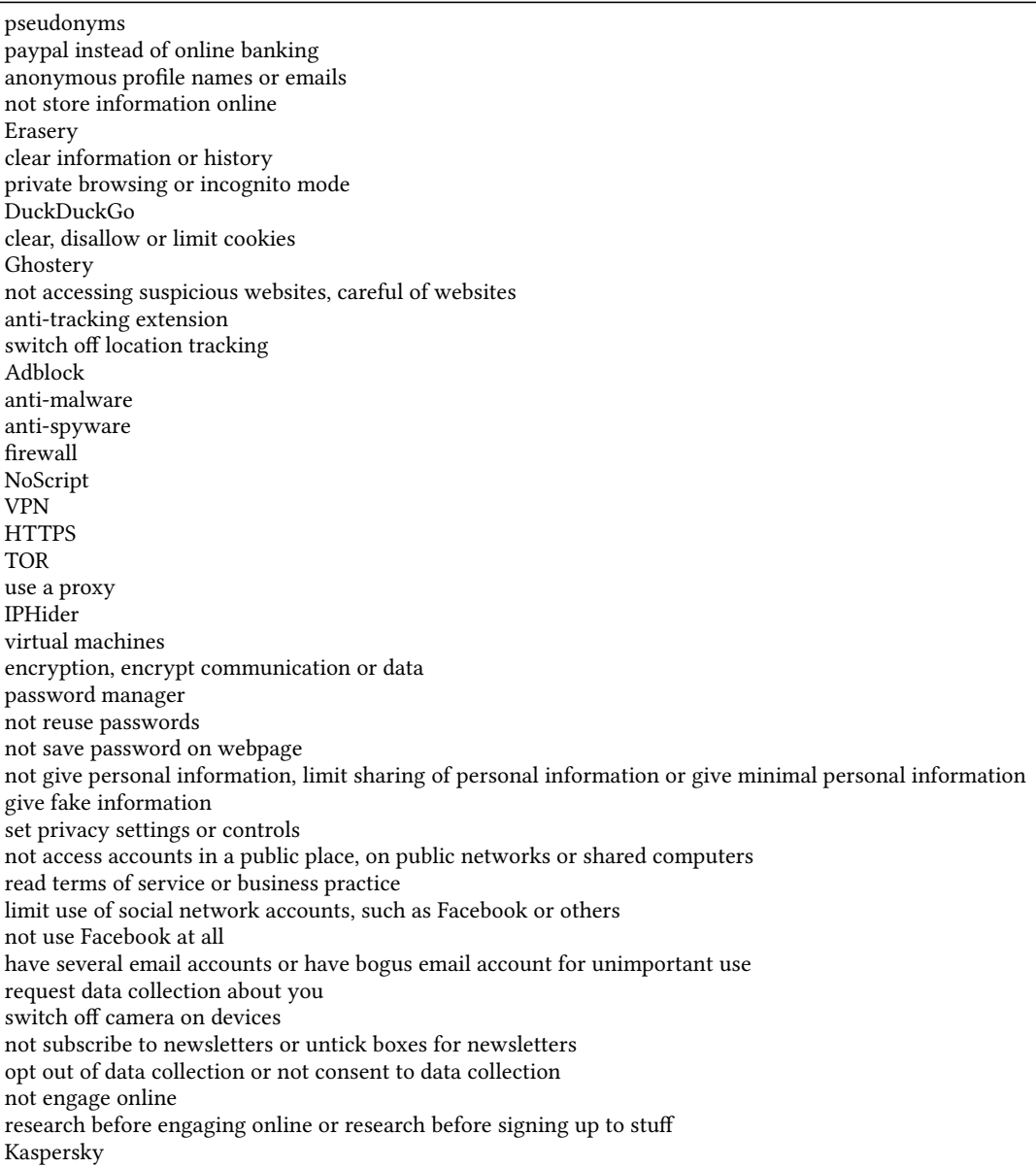 & $\begin{array}{l}\square \\
\square \\
\square \\
\square \\
\square \\
\square \\
\square \\
\square \\
\square \\
\square \\
\square \\
\square \\
\square \\
\square \\
\square \\
\square \\
\square \\
\square \\
\square \\
\square \\
\square \\
\square \\
\square \\
\square \\
\square \\
\square \\
\square \\
\square \\
\square \\
\square \\
\square \\
\square \\
\square \\
\square \\
\square \\
\square \\
\square \\
\square \\
\square \\
\square \\
\square \\
\square \\
\square\end{array}$ & $\begin{array}{l}\square \\
\square \\
\square \\
\square \\
\square \\
\square \\
\square \\
\square \\
\square \\
\square \\
\square \\
\square \\
\square \\
\square \\
\square \\
\square \\
\square \\
\square \\
\square \\
\square \\
\square \\
\square \\
\square \\
\square \\
\square \\
\square \\
\square \\
\square \\
\square \\
\square \\
\square \\
\square \\
\square \\
\square \\
\square \\
\square \\
\square \\
\square \\
\square \\
\square \\
\square \\
\square \\
\square\end{array}$ \\
\hline
\end{tabular}




\section{B STUDY 2 DATASET}

Table 13: Dataset of Privacy Methods \& Count of Usage per Country.

\begin{tabular}{|c|c|c|c|c|c|}
\hline \# & Tools/Methods & US & UK & DE & Total \\
\hline 1 & not accessing suspicious websites, careful of websites & 265 & 251 & 250 & 766 \\
\hline 2 & not give personal information or limit sharing & 259 & 242 & 256 & 757 \\
\hline 3 & set privacy settings or controls & 262 & 238 & 257 & 757 \\
\hline 4 & not subscribe to newsletters or untick boxes for newsletters & 234 & 219 & 250 & 703 \\
\hline 5 & Adblock & 235 & 197 & 262 & 694 \\
\hline 6 & clear information or history & 231 & 235 & 220 & 686 \\
\hline 7 & anti-malware & 238 & 217 & 224 & 679 \\
\hline 8 & firewall & 211 & 218 & 250 & 679 \\
\hline 9 & paypal instead of online banking & 201 & 226 & 250 & 677 \\
\hline 10 & have several email accounts & 214 & 183 & 261 & 658 \\
\hline 11 & clear, disallow or limit cookies & 225 & 198 & 219 & 642 \\
\hline 12 & opt out of data collection or not consent to data collection & 216 & 202 & 207 & 625 \\
\hline 13 & HTTPS & 209 & 172 & 237 & 618 \\
\hline 14 & not access accounts in a public place/ shared computers & 224 & 210 & 171 & 605 \\
\hline 15 & anti-spyware & 221 & 190 & 181 & 592 \\
\hline 16 & switch off camera on devices & 206 & 172 & 209 & 587 \\
\hline 17 & private browsing or incognito mode & 194 & 179 & 212 & 585 \\
\hline 18 & limit use of social network accounts & 189 & 186 & 209 & 584 \\
\hline 19 & switch off location tracking & 183 & 174 & 201 & 558 \\
\hline 20 & anonymous profile names or emails & 175 & 134 & 228 & 537 \\
\hline 21 & pseudonyms & 143 & 111 & 229 & 483 \\
\hline 22 & not reuse passwords & 162 & 148 & 158 & 468 \\
\hline 23 & not store information online & 167 & 144 & 154 & 465 \\
\hline 24 & not save password on webpage & 147 & 147 & 149 & 443 \\
\hline 25 & give fake information & 128 & 102 & 181 & 411 \\
\hline 26 & read terms of service or business practice & 154 & 147 & 100 & 401 \\
\hline 27 & password manager & 152 & 124 & 116 & 392 \\
\hline 28 & not use Facebook at all & 131 & 99 & 160 & 390 \\
\hline 29 & VPN & 99 & 86 & 157 & 342 \\
\hline 30 & encryption, encrypt communication or data & 117 & 103 & 119 & 339 \\
\hline 31 & anti-tracking extension & 93 & 64 & 126 & 283 \\
\hline 32 & not engage online & 99 & 97 & 69 & 265 \\
\hline 33 & use a proxy & 67 & 65 & 110 & 242 \\
\hline 34 & DuckDuckGo & 79 & 39 & 77 & 195 \\
\hline 35 & request data collection about you & 54 & 38 & 81 & 173 \\
\hline 36 & NoScript & 39 & 21 & 84 & 144 \\
\hline 37 & IPHider & 42 & 35 & 61 & 138 \\
\hline 38 & research before engaging online & 30 & 35 & 63 & 128 \\
\hline 39 & Kaspersky & 30 & 35 & 63 & 128 \\
\hline 40 & virtual machines & 37 & 21 & 67 & 125 \\
\hline 41 & Tor & 32 & 23 & 67 & 122 \\
\hline 42 & Ghostery & 31 & 21 & 55 & 107 \\
\hline 43 & Erasery & 27 & 30 & 37 & 94 \\
\hline
\end{tabular}

Notes: This dataset is for the $N=907$ sample, and sorted from most used to least used method. This dataset was used in the Cluster and Correspondence Analyses. 


\section{CORRESPONDENCE ANALYSIS}

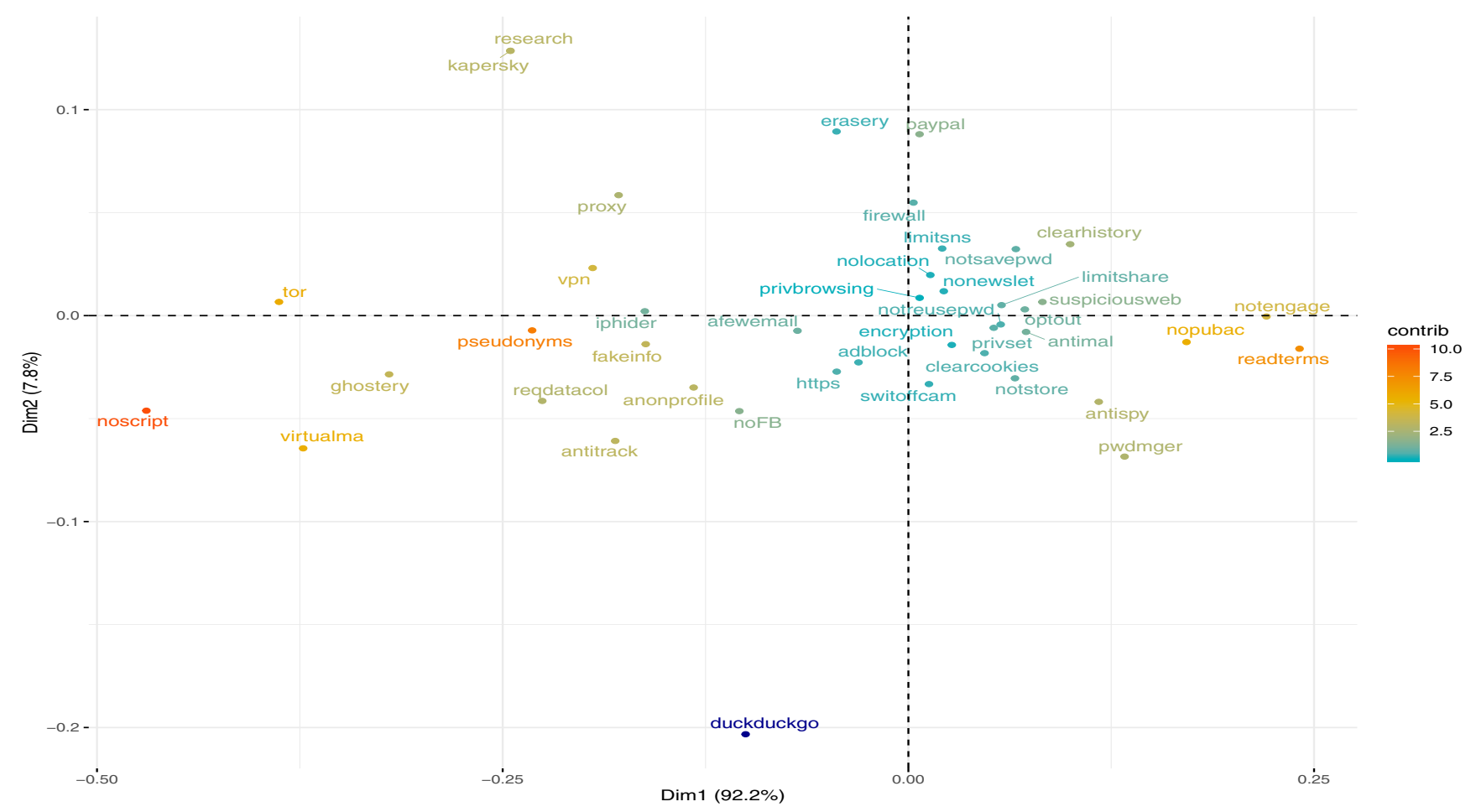

Figure 7: Contribution of Privacy Methods to Dimension 1. 


\section{STUDY 3 LISTS QUESTIONNAIRE}

The instructions provided to participants to choose between two lists of PETs and the follow-up questions are as follow:

We provide two lists of privacy technologies that can be used to protect privacy online. Please take your time to read through both lists.

List A contains: Erasery, Ghostery, Virtual Machine, Tor, NoScript, IPHider, Kaspersky, DuckDuckGo, proxy, anti-tracking extension, VPN and encryption.

List B contains: switch off location tracking, private browsing, HTTPS, anti-spyware, opt-out (of data collection), clear cookies, antimalware, clear history, Paypal, firewall, Adblock, privacy settings, pseudonyms and anonymous profile.

Which of the two lists contains the privacy methods that you most often use to protection your privacy online?

List A

List B

Please explain why you most often use methods from your selected list. Please explain why you very rarely (or not at all) use methods from the other list. [Response in 50 to 100 words]

What would support you to use methods from the other list (that is, the one that you did not select)? In particular, what would help and/or encourage you?

\section{E STUDY 3 PERCEPTION QUESTIONNAIRE SCALE ITEMS}

Table 14: Scale Items \& Internal Consistency. We provide Cronbach $\alpha$ for Adv. \& Oth.PETs where the scale was set as pairwise comparison between PET types.

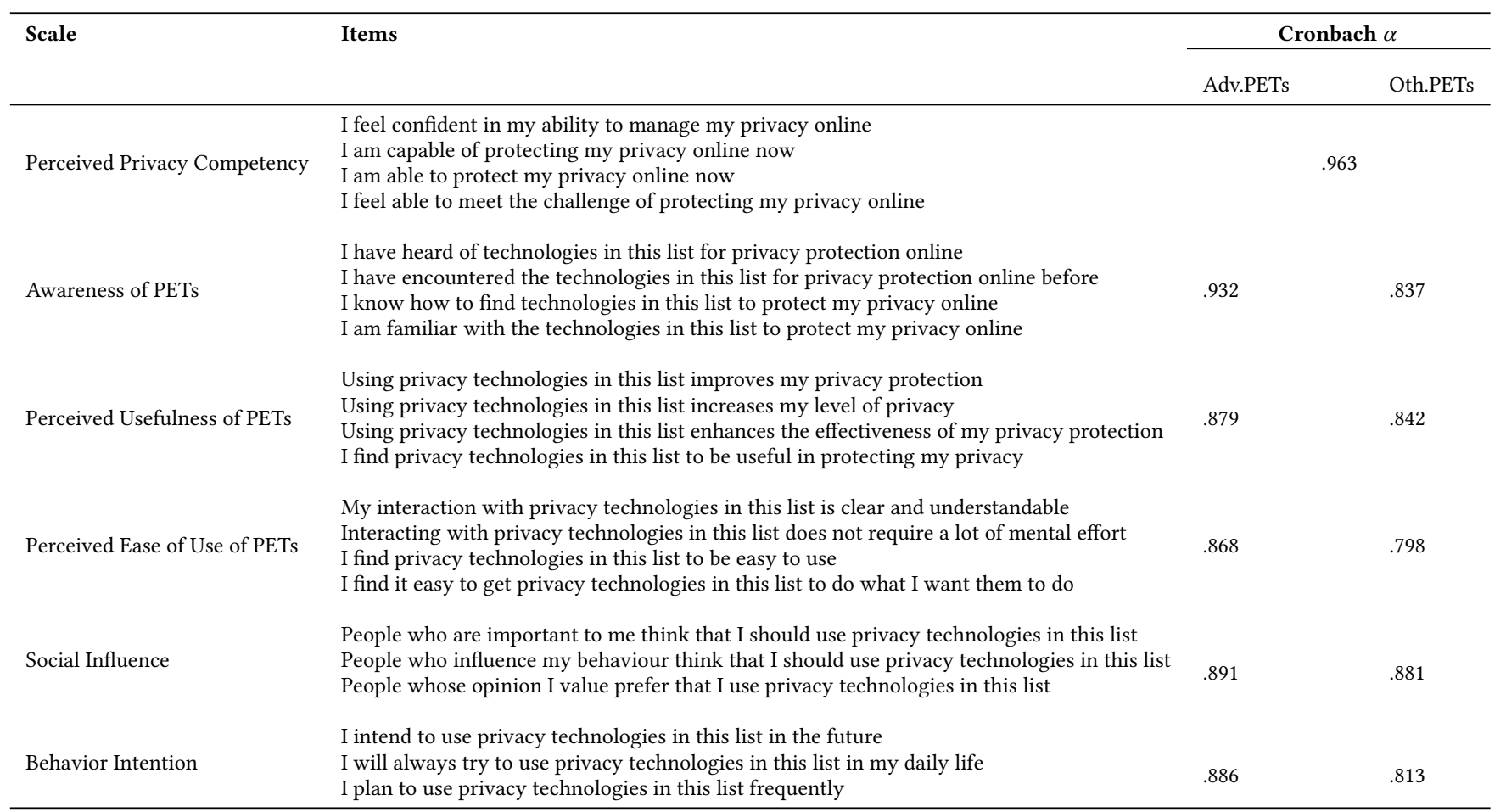




\section{F STUDY 3 CODEBOOK}

Table 15: The 47 codes in the final codebook for Study 3

\begin{tabular}{|c|c|c|c|}
\hline \multicolumn{2}{|c|}{ Q1: Reason for choosing one PET type } & \multicolumn{2}{|c|}{ Q2: Support or encouragement } \\
\hline Code & Content & Code & Content \\
\hline \multicolumn{2}{|r|}{ Effectiveness of PETs } & \multicolumn{2}{|r|}{$\begin{array}{l}\text { Privacy need } \\
\end{array}$} \\
\hline EFF01 & effective for the privacy I need, adequate, sufficient & PNE01 & if I had a bigger privacy need, extreme privacy \\
\hline EFF02 & used it and it works - so continue to use & PNE02 & needed for different specific privacy protections such as tracking prevention \\
\hline EFF03-other & everyday, regular use & PNE03 & provides more privacy than the one I use \\
\hline \multirow[t]{3}{*}{ EFF03-other } & trust, reliable, best method & & \\
\hline & & & Information or training to enhance understanding \\
\hline & Awareness of PETs & INF01 & if I know what they are/what they do \\
\hline AWA1 & familiar with, recognise, heard of, know of & INF02 & Information on how to use or training or education \\
\hline AWA2 & not familiar with, not recognise, not heard of, don't know of & INF03 & Information channel such as advertising, social media, tutorial, app \\
\hline AWA3 & aware of though advertisements & INF04-other & benefits of using, why use \\
\hline AWA4-other & advised, recommended & INF04-other & tech knowledge \\
\hline \multicolumn{2}{|r|}{ Skills needed } & \multicolumn{2}{|r|}{ Usability related supports } \\
\hline SKI01 & casual, non-tech user & USS1 & convenient \\
\hline SKI02 & training needed, need to understand or know more, technical user & USS2 & easy to use, simple, clear \\
\hline \multirow[t]{3}{*}{ SKI03-other } & someone to show me & USS3 & easy to install, setup, not many config \\
\hline & & USS4 & lightweight, not many things, easy integration \\
\hline & Usability \& cost characteristics of PET & USS5 & easy access \\
\hline USR1 & readily or easily available, builtin, presented within service & & \\
\hline USR2 & not easily or not readily available & & Social influence or social support \\
\hline USR3 & easy to use, easy to install, not much effort needed & SOC01 & social influence - if someone I know use it, or someone I trust recommends it \\
\hline USR4 & complicated to use or complicated to setup & $\mathrm{SOC} 02$ & social support - someone teaches me, someone installs it \\
\hline USR5 & convenient features & SOC03-other & neutral recommend, review, professional reputable company, accreditation \\
\hline USR6 & cost - available free & & \\
\hline USR7 & cost - expensive or have to pay for & & Miscellaneous \\
\hline USR8-other & accessibility, easy access & INC01 & if I knew they did not pose a security threat, if I trust them \\
\hline USR8-other & amount of work vs benefit, having to download/install & INC02 & free of cost, affordable \\
\hline USR8-other & annoyance, discomfort, break things & INC03 & already use both lists \\
\hline \multirow[t]{2}{*}{ USR8-other } & integrated in service & INC04-other & tradeoff with browsing or computer performance \\
\hline & Privacy needed, experience & & \\
\hline PRI01 & no need for privacy & & \\
\hline PRI02 & privacy vs hamper online experience & & \\
\hline PRI03 & provide protection, keeps me privacy/safe, increase my privacy & & \\
\hline PRI05 & use PETs from both lists & & \\
\hline PRI06-other & over the top, extreme privacy & & \\
\hline
\end{tabular}

